JOURNAL OF THE

AMERICAN MATHEMATICAL SOCIETY

Volume 16, Number 3, Pages 705-749

S 0894-0347(03)00421-1

Article electronically published on January 29, 2003

\title{
SHARP GLOBAL WELL-POSEDNESS FOR KDV AND MODIFIED KDV ON $\mathbb{R}$ AND $\mathbb{T}$
}

\author{
J. COLLIANDER, M. KEEL, G. STAFFILANI, H. TAKAOKA, AND T. TAO
}

\section{Contents}

1. Introduction 706

1.1. GWP below the conservation law 707

1.2. The operator $I$ and almost conserved quantities 708

1.3. Outline 711

1.4. Notation 712

2. Multilinear forms 712

3. Modified energies 713

4. Pointwise multiplier bounds 715

4.1. Arithmetic and calculus facts $\quad 715$

4.2. $M_{3}$ bound 715

4.3. $M_{4}$ bound. 716

4.4. $M_{5}$ bound 720

5. Quintilinear estimate on $\mathbb{R} \quad 720$

6. Global well-posedness of $\mathrm{KdV}$ on $\mathbb{R} \quad 722$

6.1. A variant local well-posedness result 723

6.2. Rescaling $\quad \frac{723}{723}$

6.3. Almost conservation $\quad 723$

6.4. Iteration 726

7. Local well-posedness of $\mathrm{KdV}$ on $\mathbb{T} \quad 727$

7.1. The $\lambda$-periodic initial value problem for $\mathrm{KdV}$

7.2. Spaces of functions of space-time 729

7.3. Linear estimates 730

7.4. Bilinear estimate 732

7.5. Contraction 737

8. Almost conservation and global well-posedness of $\mathrm{KdV}$ on $\mathbb{T} \quad 739$

Received by the editors November 7, 2001 and, in revised form, November 19, 2002.

2000 Mathematics Subject Classification. Primary 35Q53, 42B35, 37K10.

Key words and phrases. Korteweg-de Vries equation, nonlinear dispersive equations, bilinear estimates, multilinear harmonic analysis.

The first author is supported in part by N.S.F. Grant DMS 0100595.

The second author is supported in part by N.S.F. Grant DMS 9801558.

The third author is supported in part by N.S.F. Grant DMS 9800879 and by a grant from the Sloan Foundation.

The fourth author is supported in part by J.S.P.S. Grant No. 13740087.

The last author is a Clay Prize Fellow and is supported in part by grants from the Packard and Sloan Foundations.

(C)2003 American Mathematical Society 
8.1. Quintilinear estimate $\quad 739$

8.2. Rescaling $\quad 741$

8.3. Almost conservation and iteration $\quad 741$

9. Global well-posedness for modified KdV $\quad 742$

\begin{tabular}{lll} 
9.1. Defocussing case & 742 \\
\hline
\end{tabular}

9.2. Focussing case $\quad 744$

9.3. Modified $\mathrm{KdV}$ on $\mathbb{T} \quad 746$

References 747

\section{INTRODUCTION}

The initial value problem for the Korteweg-de Vries (KdV) equation,

$$
\left\{\begin{array}{l}
\partial_{t} u+\partial_{x}^{3} u+\frac{1}{2} \partial_{x} u^{2}=0, \quad u: \mathbb{R} \times[0, T] \longmapsto \mathbb{R} \\
u(0)=\phi \in H^{s}(\mathbb{R})
\end{array}\right.
$$

has been shown to be locally well-posed (LWP) for $s>-\frac{3}{4}$. Kenig, Ponce and Vega [32] extended the local-in-time analysis of Bourgain [5], valid for $s \geq 0$, to the range $s>-\frac{3}{4}$ by constructing the solution of (1.1) on a time interval $[0, \delta]$ with $\delta$ depending upon $\|\phi\|_{H^{s}(\mathbb{R})}$. Earlier results can be found in [4], 28], 23], 31], [12]. We prove here that these solutions exist for $t$ in an arbitrary time interval $[0, T]$ thereby establishing global well-posedness (GWP) of (1.1) in the full range $s>-\frac{3}{4}$. The corresponding periodic $\mathbb{R}$-valued initial value problem for $\mathrm{KdV}$

$$
\left\{\begin{array}{c}
\partial_{t} u+\partial_{x}^{3} u+\frac{1}{2} \partial_{x} u^{2}=0, \quad u: \mathbb{T} \times[0, T] \longmapsto \mathbb{R} \\
u(0)=\phi \in H^{s}(\mathbb{T})
\end{array}\right.
$$

is known 32] to be locally well-posed for $s \geq-\frac{1}{2}$. These local-in-time solutions are also shown to exist on an arbitrary time interval. Bourgain established [9] global well-posedness of (1.2) for initial data having (small) bounded Fourier transform. The argument in [9] uses the complete integrability of KdV. Analogous globalizations of the best known local-in-time theory for the focussing and defocussing modified $\mathrm{KdV}(\mathrm{mKdV})$ equations $\left(u^{2}\right.$ in (1.1), (1.2) replaced by $-u^{3}$ and $u^{3}$, respectively) are also obtained in the periodic $\left(s \geq \frac{1}{2}\right)$ and real line $\left(s>\frac{1}{4}\right)$ settings.

The local-in-time theory globalized here is sharp (at least up to certain endpoints) in the scale of $L^{2}$-based Sobolev spaces $H^{s}$. Indeed, recent examples [33] of Kenig, Ponce and Vega (see also [2], 3]) reveal that focussing $\mathrm{mKdV}$ is ill-posed for $s<\frac{1}{4}$ and that $\mathbb{C}$-valued $\operatorname{KdV}(u: \mathbb{R} \times[0, T] \longmapsto \mathbb{C})$ is ill-posed for $s<-\frac{3}{4}$. (The local theory in 32 adapts easily to the $\mathbb{C}$-valued situation.) A similar failure of local well-posedness below the endpoint regularities for the defocusing modified $\mathrm{KdV}$ and the $\mathbb{R}$-valued $\mathrm{KdV}$ has been established [13] by Christ, Colliander and Tao. The fundamental bilinear estimate used to prove the local well-posedness result on the line was shown to fail for $s \leq-\frac{3}{4}$ by Nakanishi, Takaoka and Tsutsumi [45]. Nevertheless, a conjugation of the $H^{\frac{1}{4}}$ local well-posedness theory for defocusing mKdV using the Miura transform established [13] a local well-posedness result for $\mathrm{KdV}$ at the endpoint $H^{-\frac{3}{4}}(\mathbb{R})$. Global well-posedness of $\mathrm{KdV}$ at the $-\frac{3}{4}$ endpoint and for $\mathrm{mKdV}$ in $H^{\frac{1}{4}}$ remain open problems. 
1.1. GWP below the conservation law. $\mathbb{R}$-valued solutions of KdV satisfy $L^{2}$ conservation: $\|u(t)\|_{L^{2}}=\|\phi\|_{L^{2}}$. Consequently, a local well-posedness result with the existence lifetime determined by the size of the initial data in $L^{2}$ may be iterated to prove global well-posedness of $\mathrm{KdV}$ for $L^{2}$ data [5]. What happens to solutions of $\mathrm{KdV}$ which evolve from initial data which are less regular than $L^{2}$ ? Bourgain observed, in a context [8] concerning very smooth solutions, that the nonlinear Duhamel term may be smoother than the initial data. This observation was exploited [8], using a decomposition of the evolution of the high and low frequency parts of the initial data, to prove polynomial-in-time bounds for global solutions of certain nonlinear Schrödinger (NLS) and nonlinear wave (NLW) equations. In [10], Bourgain introduced a general high/low frequency decomposition argument to prove that certain NLS and NLW equations were globally well-posed below $H^{1}$, the natural regularity associated with the conserved Hamiltonian. Subsequently, Bourgain's high/low method has been applied to prove global well-posedness below the natural regularity of the conserved quantity in various settings [20, 50, [48, [34], including $\mathrm{KdV}$ [18] on the line. A related argument-directly motivated by Bourgain's work-appeared in [29, 30] where the presence of derivatives in the nonlinearities leaves a Duhamel term which cannot be shown to be smoother than the initial data. Global rough solutions for these equations are constructed with a slightly different use of the original conservation law (see below).

We summarize the adaptation [18] of the high/low method to construct a solution of (1.1) for rough initial data. The task is to construct the global solution of (1.1) evolving from initial data $\phi \in H^{s}(\mathbb{R})$ for $s_{0}<s<0$ with $-3 / 4 \ll s_{0} \lesssim 0$. The argument [18] accomplishes this task for initial data in a subset of $H^{s}(\mathbb{R})$ consisting of functions with relatively small low frequency components. Split the data $\phi=\phi_{0}+\psi_{0}$ with $\widehat{\phi_{0}}(k)=\chi_{[-N, N]}(k) \widehat{\phi}(k)$, where $N=N(T)$ is a parameter to be determined. The low frequency part $\phi_{0}$ of $\phi$ is in $L^{2}(\mathbb{R})$ (in fact $\phi_{0} \in H^{s}$ for all $s$ ) with a big norm while the high frequency part $\psi_{0}$ is the tail of an $H^{s}(\mathbb{R})$ function and is therefore small (with large $N$ ) in $H^{\sigma}(\mathbb{R})$ for any $\sigma<s$. The low frequencies are evolved according to KdV: $\phi_{0} \longmapsto u_{0}(t)$. The high frequencies evolve according to a "difference equation" which is selected so that the sum of the resulting high frequency evolution, $\psi_{0} \longmapsto v_{0}(t)$ and the low frequency evolution solves (1.1). The key step is to decompose $v_{0}(t)=S(t) \psi_{0}+w_{0}(t)$, where $S(t)$ is the solution operator of the Airy equation. For the selected class of rough initial data mentioned above, one can then prove that $w_{0} \in L^{2}(\mathbb{R})$ and has a small (depending upon $N$ ) $L^{2}$ norm. Then an iteration of the local-in-time theory advances the solution to a long (depending on $N$ ) time interval. An appropriate choice of $N$ completes the construction.

The nonlinear Duhamel term for the "difference equation" mentioned above is

$$
w_{0}(t)=\int_{0}^{t} S\left(t-t^{\prime}\right)\left(\left[v_{0}^{2}\left(t^{\prime}\right)+2 u_{0}\left(t^{\prime}\right) v_{0}\left(t^{\prime}\right)\right]\right) d t^{\prime}
$$

The local well-posedness machinery [5], 32] allows us to prove that $w_{0}(t) \in L^{2}(\mathbb{R})$ if we have the extra smoothing bilinear estimate

$$
\left\|\partial_{x}(u v)\right\|_{X_{0, b-1}} \lesssim\|u\|_{X_{s, b}}\|v\|_{X_{s, b}}, \quad s<0, b=\frac{1}{2}+,
$$

with the space $X_{s, b}$ defined below (see (1.11)). The estimate (1.3) is valid for functions $u, v$ such that $\widehat{u}, \widehat{v}$ are supported outside $\{|k| \leq 1\}$, in the range $-\frac{3}{8}<s$ 
18], [15. The estimate (1.3) fails for $s<-\frac{3}{8}$ and this places an intrinsic limitation on how far the high/low frequency decomposition technique may be used to extend GWP for rough initial data. Also, (1.3) fails without some assumptions on the low frequencies of $u$ and $v$, hence the initial data considered in the high/low argument of [18. We showed that the low frequency issue may indeed be circumvented in [15] by proving (1.1) is GWP in $H^{s}(\mathbb{R}), s>-\frac{3}{10}$. The approach in [15] does not rely on showing the nonlinear Duhamel term has regularity at the level of the conservation law. We review this approach now and motivate the nontrivial improvements of that argument leading to sharp global regularity results for (1.1) and (1.2).

1.2. The operator $I$ and almost conserved quantities. Global well-posedness follows from (an iteration of) local well-posedness (results) provided the successive local-in-time existence intervals cover an arbitrary time interval $[0, T]$. The length of the local-in-time existence interval is controlled from below by the size of the initial data in an appropriate norm. A natural approach to global well-posedness in $H^{s}$ is to establish upper bounds on $\|u(t)\|_{H^{s}}$ for solutions $u(t)$ which are strong enough to prove that $[0, T]$ may be covered by iterated local existence intervals. We establish appropriate upper bounds to carry out this general strategy by constructing almost conserved quantities and rescaling. The rescaling exploits the subcritical nature of the $\mathrm{KdV}$ initial value problem (but introduces technical issues in the treatment of the periodic problem). The almost conserved quantities are motivated by the following discussion of the $L^{2}$ conservation property of solutions of $\mathrm{KdV}$.

Consider the following Fourier proof that $\|u(t)\|_{L^{2}}=\|\phi\|_{L^{2}} \forall t \in \mathbb{R}$. By Plancherel,

$$
\|u(t)\|_{L^{2}}^{2}=\int \widehat{u}(\xi) \widehat{\widehat{u}}(\xi) d \xi
$$

where

$$
\widehat{u}(\xi)=\int e^{-i x \xi} u(x) d x
$$

is the (spatial) Fourier transform. Fourier transform properties imply

$$
\int \widehat{u}(\xi) \bar{u}(\xi) d \xi=\int \widehat{u}(\xi) \widehat{\bar{u}}(-\xi) d \xi=\int_{\xi_{1}+\xi_{2}=0} \widehat{u}\left(\xi_{1}\right) \widehat{\bar{u}}\left(\xi_{2}\right) .
$$

Since we are assuming $u$ is $\mathbb{R}$-valued, we may replace $\widehat{\bar{u}}\left(\xi_{2}\right)$ by $\widehat{u}\left(\xi_{2}\right)$. Hence,

$$
\|u(t)\|_{L^{2}}^{2}=\int_{\xi_{1}+\xi_{2}=0} \widehat{u}\left(\xi_{1}\right) \widehat{u}\left(\xi_{2}\right)
$$

We apply $\partial_{t}$, and we use symmetry and the equation to find

$$
\partial_{t}\left(\|u(t)\|_{L^{2}}^{2}\right)=2 i \int_{\xi_{1}+\xi_{2}=0} \xi_{1}^{3} \widehat{u}\left(\xi_{1}\right) \widehat{u}\left(\xi_{2}\right)-i \int_{\xi_{1}+\xi_{2}=0} \xi_{1} \widehat{u^{2}}\left(\xi_{1}\right) \widehat{u}\left(\xi_{2}\right) .
$$

The first expression is symmetric under the interchange of $\xi_{1}$ and $\xi_{2}$ so $\xi_{1}^{3}$ may be replaced by $\frac{1}{2}\left(\xi_{1}^{3}+\xi_{2}^{3}\right)$. Since we are integrating on the set where $\xi_{1}+\xi_{2}=0$, the

\footnotetext{
${ }^{1}$ This argument was known previously; see a similar argument in [27].
} 
integrand is zero and this term vanishes. Calculating $\widehat{u^{2}}(\xi)=\int_{\xi=\xi_{1}+\xi_{2}} \widehat{u}\left(\xi_{1}\right) \widehat{u}\left(\xi_{2}\right)$, the remaining term may be rewritten

$$
-i \int_{\xi_{1}+\xi_{2}+\xi_{3}=0}\left[\xi_{1}+\xi_{2}\right] \widehat{u}\left(\xi_{1}\right) \widehat{u}\left(\xi_{2}\right) \widehat{u}\left(\xi_{3}\right) .
$$

On the set where $\xi_{1}+\xi_{2}+\xi_{3}=0, \xi_{1}+\xi_{2}=-\xi_{3}$ which we symmetrize to replace $\xi_{1}+\xi_{2}$ in (1.4) by $-\frac{1}{3}\left(\xi_{1}+\xi_{2}+\xi_{3}\right)$ and this term vanishes as well. Summarizing, we have found that $\mathbb{R}$-valued solutions $u(t)$ of $\mathrm{KdV}$ satisfy

$$
\begin{aligned}
\partial_{t}\left(\|u(t)\|_{L^{2}}^{2}\right)= & -i \int_{\xi_{1}+\xi_{2}=0}\left(\xi_{1}^{3}+\xi_{2}^{3}\right) \widehat{u}\left(\xi_{1}\right) \widehat{u}\left(\xi_{2}\right) \\
& +\frac{i}{3} \int_{\xi_{1}+\xi_{2}+\xi_{3}=0}\left(\xi_{1}+\xi_{2}+\xi_{3}\right) \widehat{u}\left(\xi_{1}\right) \widehat{u}\left(\xi_{2}\right) \widehat{u}\left(\xi_{3}\right)
\end{aligned}
$$

and both integrands on the right side vanish.

We introduce the (spatial) Fourier multiplier operator $I u$ defined via

$$
\widehat{I u}(\xi)=m(\xi) \widehat{u}(\xi)
$$

with an arbitrary $\mathbb{C}$-valued multiplier $m$. A formal imitation of the Fourier proof of $L^{2}$-mass conservation above reveals that for $\mathbb{R}$-valued solutions of $\mathrm{KdV}$ we have

$$
\begin{gathered}
\partial_{t}\left(\|I u(t)\|_{L^{2}}^{2}\right)=-\frac{i}{2} \int_{\xi_{1}+\xi_{2}=0}\left[m\left(\xi_{1}\right) \bar{m}\left(\xi_{1}\right)+m\left(\xi_{2}\right) \bar{m}\left(\xi_{2}\right)\right]\left\{\xi_{1}^{3}+\xi_{2}^{3}\right\} \widehat{u}\left(\xi_{1}\right) \widehat{u}\left(\xi_{2}\right) \\
\quad+\frac{i}{6} \int_{\xi_{1}+\xi_{2}+\xi_{3}=0} \sum_{j=1}^{3}\left[m\left(-\xi_{j}\right) \bar{m}\left(-\xi_{j}\right)+m\left(\xi_{j}\right) \bar{m}\left(\xi_{j}\right)\right] \xi_{j} \widehat{u}\left(\xi_{1}\right) \widehat{u}\left(\xi_{2}\right) \widehat{u}\left(\xi_{3}\right) .
\end{gathered}
$$

The term arising from the dispersion cancels since $\xi_{1}^{3}+\xi_{2}^{3}=0$ on the set where $\xi_{1}+\xi_{2}=0$. The remaining trilinear term can be analyzed under various assumptions on the multiplier $m$ giving insight into the time behavior of $\|I u(t)\|_{L^{2}}$. Moreover, the flexibility in our choice of $m$ may allow us to observe how the conserved $L^{2}$ mass is moved around in frequency space during the KdV evolution.

Remark 1.1. Our use of the multiplier $m$ to localize the $L^{2}$ mass in frequency space is analogous to the use of cutoff functions to spatially localize the conserved density in physical space. In that setting, the underlying conservation law $\partial_{t}($ conserved density $)+\partial_{x}($ flux $)=0$ is multiplied by a cutoff function. The localized flux term is no longer a perfect derivative and is then estimated, sometimes under an appropriate choice of the cutoff, to obtain bounds on the spatially localized energy.

Consider now the problem of proving well-posedness of (1.1) or (1.2), with $s<0$, on an arbitrary time interval $[0, T]$. We define a spatial Fourier multiplier operator $I$ which acts like the identity on low frequencies and like a smoothing operator of order $|s|$ on high frequencies by choosing a smooth monotone multiplier satisfying

$$
m(\xi)=\left\{\begin{array}{cc}
1, & |\xi|<N \\
N^{-s}|\xi|^{s}, & |\xi|>2 N
\end{array}\right.
$$

The parameter $N$ marks the transition from low to high frequencies. When $N=1$, the operator $I$ is essentially the integration (since $s<0$ ) operator $D^{s}$. When 
$N=\infty, I$ acts like the identity operator. Note that $\|I \phi\|_{L^{2}}$ is bounded if $\phi \in$ $H^{s}$. We prove a variant local well-posedness result which shows the length of the local existence interval $[0, \delta]$ for (1.1) or (1.2) may be bounded from below by $\|I \phi\|_{L^{2}}^{-\alpha}, \alpha>0$, for an appropriate range of the parameter $s$. The basic idea is then to bound the trilinear term in (1.6) to prove, for a particular small $\beta>0$, that

$$
\sup _{t \in[0, \delta]}\|I u(t)\|_{L^{2}} \leq\|I u(0)\|_{L^{2}}+c N^{-\beta}\|\operatorname{Iu}(0)\|_{L^{2}}^{3} .
$$

If $N$ is huge, (1.7) shows there is at most a tiny increment in $\|I u(t)\|_{L^{2}}$ as $t$ evolves from 0 to $\delta$. An iteration of the local theory under appropriate parameter choices gives global well-posedness in $H^{s}$ for certain $s<0$.

The strategy just described is enhanced with two extra ingredients: a multilinear correction technique and rescaling. The correction technique shows that, up to errors of smaller order in $N$, the trilinear term in (1.6) may be replaced by a quintilinear term improving (1.7) to

$$
\sup _{t \in[0, \delta]}\|I u(t)\|_{L^{2}} \leq\|I u(0)\|_{L^{2}}+c N^{-3-\frac{3}{4}+\epsilon}\|I u(0)\|_{L^{2}}^{5},
$$

where $\epsilon$ is tiny. The rescaling argument reduces matters to initial data $\phi$ of fixed size: $\|I \phi\|_{L^{2}} \sim \epsilon_{0} \ll 1$. In the periodic setting, the rescaling we use forces us to track the dependence upon the spatial period in the local well-posedness theory [5], 32 .

The main results obtained here are:

Theorem 1. The $\mathbb{R}$-valued initial value problem (1.1) is globally well-posed for initial data $\phi \in H^{s}(\mathbb{R}), s>-\frac{3}{4}$.

Theorem 2. The $\mathbb{R}$-valued periodic initial value problem (1.2) is globally well-posed for initial data $\phi \in H^{s}(\mathbb{T}), s \geq-\frac{1}{2}$.

Theorem 3. The $\mathbb{R}$-valued initial value problem for modified $K d V$ (9.1) (focussing or defocussing) is globally well-posed for initial data $\phi \in H^{s}(\mathbb{R}), s>\frac{1}{4}$.

Theorem 4. The $\mathbb{R}$-valued periodic initial value problem for modified $K d V$ (focussing or defocussing) is globally well-posed for initial data $\phi \in H^{s}(\mathbb{T}), s \geq \frac{1}{2}$.

The infinite-dimensional symplectic nonsqueezing machinery developed by $\mathrm{S}$. Kuksin [36] identifies $H^{-\frac{1}{2}}(\mathbb{T})$ as the Hilbert Darboux (symplectic) phase space for $\mathrm{KdV}$. We anticipate that Theorem 3 will be useful in adapting these ideas to the $\mathrm{KdV}$ context. The main remaining issue is an approximation of the $\mathrm{KdV}$ flow using finite-dimensional Hamiltonian flows analogous to that obtained by Bourgain [6] in the NLS setting. We plan to address this topic in a forthcoming paper.

We conclude this subsection with a discussion culminating in a table which summarizes the well-posedness theory in Sobolev ${ }^{2}$ spaces $H^{s}$ for the polynomial generalized $\mathrm{KdV}$ equations. The initial value problem

$$
\left\{\begin{array}{c}
\partial_{t} u+\partial_{x}^{3} u \pm \frac{1}{k} \partial_{x} u^{k}=0, \quad u: \mathbb{R} \times[0, T] \longmapsto \mathbb{R} \\
u(0)=\phi \in H^{s}(\mathbb{R})
\end{array}\right.
$$

has the associated Hamiltonian

$$
H[u]=\int \frac{1}{2} u_{x}^{2} \mp \frac{1}{k(k+1)} u^{k+1} d x .
$$

\footnotetext{
${ }^{2}$ There are results, e.g. 9], in function spaces outside the $L^{2}$-based Sobolev scale.
} 
The replacement $u \longmapsto-u$ shows that the \pm choice is irrelevant when $k$ is even, but, when $k$ is odd there are two distinct cases in (1.9): + is called focussing and - is called defocussing. The usefulness of the Hamiltonian in controlling the $H^{1}$ norm can depend upon the $\mp$ choice in 1.10).

We now summarize the well-posedness theory for the generalized KdV equations. The notation $\mathrm{D}$ and $\mathrm{F}$ in Table 1 refers to the defocussing and focussing cases. We highlight with the notation ?? some issues which are not yet resolved (as far as we are aware).

TABle 1. $\mathbb{R}$-Valued Generalized KdV on $\mathbb{R}$ Well-posedness Summary Table

\begin{tabular}{||c|c|c|c|c||}
\hline $\mathbf{k}$ & Scaling & Ill-posed & L.W.P. & G.W.P. \\
\hline 2 & $-\frac{3}{2}$ & $s<-\frac{3}{4},[13]$ & $s \geq-\frac{3}{4},[32] ;[13]$ & $s>-\frac{3}{4}$ \\
\hline 3 & $-\frac{1}{2}$ & $s<\frac{1}{4}$, F:[33], D:[13] & $s \geq \frac{1}{4},[3]$ & $s>\frac{1}{4}$ \\
\hline 4 & $-\frac{1}{6}$ & $s<-\frac{1}{6},[33]$ & $s>-\frac{1}{6},[25]$ & $s \geq 0$ \\
\hline 5 & 0 & F: $s<0,[33]$, D: ?? & $s \geq 0,[31]$ & D: $s>\frac{3}{4}[21]$ \\
\cline { 4 - 5 } & & & & F: $s>\frac{3}{4}, L^{2}$ small [21] \\
\cline { 5 - 6 } & & & & F: big $L^{2}$ blows up [38] \\
\cline { 4 - 5 }$k \geq 6$ & $s_{k}=\frac{1}{2}-\frac{2}{k}$ & F: $s<s_{k},[33] ;$ D: ?? & $s \geq s_{k},[31]$ & D: $s \geq 1$, F: small $H^{1}$ \\
\cline { 4 - 5 } & & & F: big $H^{1}$ blows up ?? \\
\hline
\end{tabular}

Our results here and elsewhere [16], 14], [17] suggest that local well-posedness implies global well-posedness in subcritical dispersive initial value problems. In particular, we believe our methods will extend to prove GWP of $\mathrm{mKdV}$ in $H^{\frac{1}{4}}(\mathbb{R})$ and $\mathrm{KdV}$ in $H^{-\frac{3}{4}}(\mathbb{R})$ and also extend the GWP intervals in the cases $k \geq 4$. However, our results rely on the fact that we are considering the $\mathbb{R}$-valued KdV equation and, due to a lack of conservation laws, we do not know if the local results for the $\mathbb{C}$-valued KdV equation may be similarly globalized. An adaptation of techniques from [13] may provide ill-posedness results in the higher power defocussing cases. Blow up in the focussing supercritical ( $k \geq 6$ or, more generally, $k \in \mathbb{R}$ with $k>5$ ) is expected to occur but no rigorous results in this direction have been so far obtained [39].

1.3. Outline. Sections 2 and 3 describe the multilinear correction technique which generates modified energies. Section 4 establishes useful pointwise upper bounds on certain multipliers arising in the multilinear correction procedure. These upper bounds are combined with a quintilinear estimate, in the $\mathbb{R}$ setting, to prove the bulk of (1.8) in Section 5. Section 6 contains the variant local well-posedness result and the proof of global well-posedness for (1.1) in $H^{s}(\mathbb{R}), s>-\frac{3}{4}$. We next consider the periodic initial value problem (1.2) with period $\lambda$. Section 7 extends the local well-posedness theory for (1.2) to the $\lambda$-periodic setting. Section 8 proves global well-posedness of (1.2) in $H^{s}(\mathbb{T}), s \geq-\frac{1}{2}$. The last section exploits Miura's 
transform to prove the corresponding global well-posedness results for the focussing and defocussing modified $\mathrm{KdV}$ equations.

1.4. Notation. We will use $c, C$ to denote various time independent constants, usually depending only upon $s$. In case a constant depends upon other quantities, we will try to make that explicit. We use $A \lesssim B$ to denote an estimate of the form $A \leq C B$. Similarly, we will write $A \sim B$ to mean $A \lesssim B$ and $B \lesssim A$. To avoid an issue involving a logarithm, we depart from standard practice and write $\langle k\rangle=2+|k|$. The notation $a+$ denotes $a+\epsilon$ for an arbitrarily small $\epsilon$. Similarly, $a-$ denotes $a-\epsilon$. We will make frequent use of the two-parameter spaces $X_{s, b}(\mathbb{R} \times \mathbb{R})$ with norm

$$
\|u\|_{X_{s, b}}=\left(\iint\langle\xi\rangle^{2 s}\left\langle\tau-\xi^{3}\right\rangle^{2 b}|\widehat{u}(\xi, \tau)|^{2} d \xi d \tau\right)^{\frac{1}{2}} .
$$

For any time interval $I$, we define the restricted spaces $X_{s, b}(R \times I)$ by the norm

$$
\|u\|_{X_{s, b}(\mathbb{R} \times I)}=\inf \left\{\|U\|_{X_{s, b}}:\left.U\right|_{\mathbb{R} \times I}=u\right\} .
$$

These spaces were first used to systematically study nonlinear dispersive wave problems by Bourgain 5. Klainerman and Machedon 37] used similar ideas in their study of the nonlinear wave equation. The spaces appeared earlier in a different setting in the works [46, 1] of Rauch, Reed, and M. Beals. We will systematically ignore constants involving $\pi$ in the Fourier transform, except in Section 7. Other notation is introduced during the developments that follow.

\section{Multilinear Forms}

In this section, we introduce notation for describing certain multilinear operators; see for example [4], 40. Bilinear versions of these operators will generate a sequence of almost conserved quantities involving higher order multilinear corrections.

Definition 1. A $k$-multiplier is a function $m: \mathbb{R}^{k} \longmapsto \mathbb{C}$. A $k$-multiplier is symmetric if $m\left(\xi_{1}, \xi_{2}, \ldots, \xi_{k}\right)=m\left(\sigma\left(\xi_{1}, \xi_{2}, \ldots, \xi_{k}\right)\right)$ for all $\sigma \in S_{k}$, the group of all permutations on $k$ objects. The symmetrization of a $k$-multiplier $m$ is the multiplier

$$
[m]_{s y m}\left(\xi_{1}, \xi_{2}, \ldots, \xi_{k}\right)=\frac{1}{k !} \sum_{\sigma \in S_{k}} m\left(\sigma\left(\xi_{1}, \xi_{2}, \ldots, \xi_{k}\right)\right) .
$$

The domain of $m$ is $\mathbb{R}^{k}$; however, we will only be interested in $m$ on the hyperplane $\xi_{1}+\cdots+\xi_{k}=0$.

Definition 2. A $k$-multiplier generates a $k$-linear functional or $k$-form acting on $k$ functions $u_{1}, \ldots, u_{k}$,

$$
\Lambda_{k}\left(m ; u_{1}, \ldots, u_{k}\right)=\int_{\xi_{1}+\cdots+\xi_{k}=0} m\left(\xi_{1}, \ldots, \xi_{k}\right) \widehat{u_{1}}\left(\xi_{1}\right) \cdots \widehat{u_{k}}\left(\xi_{k}\right) .
$$

We will often apply $\Lambda_{k}$ to $k$ copies of the same function $u$ in which case the dependence upon $u$ may be suppressed in the notation: $\Lambda_{k}(m ; u, \ldots, u)$ may simply be written $\Lambda_{k}(m)$.

If $m$ is symmetric, then $\Lambda_{k}(m)$ is a symmetric $k$-linear functional. 
As an example, suppose that $u$ is an $\mathbb{R}$-valued function. We calculate $\|u\|_{L^{2}}^{2}=$ $\int \widehat{u}(\xi) \overline{\widehat{u}}(\xi) d \xi=\int_{\xi_{1}+\xi_{2}=0} \widehat{u}\left(\xi_{1}\right) \widehat{u}\left(\xi_{2}\right)=\Lambda_{2}(1)$.

The time derivative of a symmetric $k$-linear functional can be calculated explicitly if we assume that the function $u$ satisfies a particular PDE. The following statement may be directly verified by using the KdV equation.

Proposition 1. Suppose u satisfies the KdV equation (1.1) and that $m$ is a symmetric k-multiplier. Then

$$
\frac{d}{d t} \Lambda_{k}(m)=\Lambda_{k}\left(m \alpha_{k}\right)-i \frac{k}{2} \Lambda_{k+1}\left(m\left(\xi_{1}, \ldots, \xi_{k-1}, \xi_{k}+\xi_{k+1}\right)\left\{\xi_{k}+\xi_{k+1}\right\}\right),
$$

where

$$
\alpha_{k}=i\left(\xi_{1}^{3}+\cdots+\xi_{k}^{3}\right) .
$$

Note that the second term in (2.3) may be symmetrized.

\section{Modified EnERGies}

Let $m: \mathbb{R} \longmapsto \mathbb{R}$ be an arbitrary even $\mathbb{R}$-valued 1-multiplier and define the associated operator by

$$
\widehat{I f}(\xi)=m(\xi) \widehat{f}(\xi) .
$$

We define the modified energy $E_{I}^{2}(t)$ by

$$
E_{I}^{2}(t)=\|I u(t)\|_{L^{2}}^{2} .
$$

The name "modified energy" is in part justified since in case $m=1, E_{I}^{2}(t)=$ $\|u(t)\|_{L^{2}}^{2}$. We will show later that for $m$ of a particular form, certain modified energies enjoy an almost conservation property. By Plancherel and the fact that $m$ and $u$ are $\mathbb{R}$-valued,

Using (2.3), we have

$$
E_{I}^{2}(t)=\Lambda_{2}\left(m\left(\xi_{1}\right) m\left(\xi_{2}\right)\right) \text {. }
$$

$$
\frac{d}{d t} E_{I}^{2}(t)=\Lambda_{2}\left(m\left(\xi_{1}\right) m\left(\xi_{2}\right) \alpha_{2}\right)-i \Lambda_{3}\left(m\left(\xi_{1}\right) m\left(\xi_{2}+\xi_{3}\right)\left\{\xi_{2}+\xi_{3}\right\}\right) .
$$

The first term vanishes. We symmetrize the remaining term to get

$$
\frac{d}{d t} E_{I}^{2}(t)=\Lambda_{3}\left(-i\left[m\left(\xi_{1}\right) m\left(\xi_{2}+\xi_{3}\right)\left(\xi_{2}+\xi_{3}\right)\right]_{\text {sym }}\right) .
$$

Note that the time derivative of $E_{I}^{2}(t)$ is a 3-linear expression. Let us denote

$$
M_{3}\left(\xi_{1}, \xi_{2}, \xi_{3}\right)=-i\left[m\left(\xi_{1}\right) m\left(\xi_{2}+\xi_{3}\right)\left\{\xi_{2}+\xi_{3}\right\}\right]_{\text {sym }} .
$$

Observe that if $m=1$, the symmetrization results in $M_{3}=c\left(\xi_{1}+\xi_{2}+\xi_{3}\right)$. This reproduces the Fourier proof of $L^{2}$-mass conservation from the introduction.

Form the new modified energy

$$
E_{I}^{3}(t)=E_{I}^{2}(t)+\Lambda_{3}\left(\sigma_{3}\right)
$$

where the symmetric 3-multiplier $\sigma_{3}$ will be chosen momentarily to achieve a cancellation. Applying (2.3) gives

$$
\frac{d}{d t} E_{I}^{3}(t)=\Lambda_{3}\left(M_{3}\right)+\Lambda_{3}\left(\sigma_{3} \alpha_{3}\right)+\Lambda_{4}\left(-i \frac{3}{2} \sigma_{3}\left(\xi_{1}, \xi_{2}, \xi_{3}+\xi_{4}\right)\left\{\xi_{3}+\xi_{4}\right\}\right)
$$


We choose

$$
\sigma_{3}=-\frac{M_{3}}{\alpha_{3}}
$$

to force the two $\Lambda_{3}$ terms in (3.4) to cancel. With this choice, the time derivative of $E_{I}^{3}(t)$ is a 4-linear expression $\Lambda_{4}\left(M_{4}\right)$ where

$$
M_{4}\left(\xi_{1}, \xi_{2}, \xi_{3}, \xi_{4}\right)=-i \frac{3}{2}\left[\sigma_{3}\left(\xi_{1}, \xi_{2}, \xi_{3}+\xi_{4}\right)\left\{\xi_{3}+\xi_{4}\right\}\right]_{\text {sym }} .
$$

Upon defining

$$
E_{I}^{4}(t)=E_{I}^{3}(t)+\Lambda_{4}\left(\sigma_{4}\right)
$$

with

$$
\sigma_{4}=-\frac{M_{4}}{\alpha_{4}}
$$

we obtain

$$
\frac{d}{d t} E_{I}^{4}(t)=\Lambda_{5}\left(M_{5}\right)
$$

where

$$
M_{5}\left(\xi_{1}, \ldots, \xi_{5}\right)=-2 i\left[\sigma_{4}\left(\xi_{1}, \xi_{2}, \xi_{3}, \xi_{4}+\xi_{5}\right)\left\{\xi_{4}+\xi_{5}\right\}\right]_{s y m} .
$$

This process can clearly be iterated to generate $E_{I}^{n}$ satisfying

$$
\frac{d}{d t} E_{I}^{n}(t)=\Lambda_{n+1}\left(M_{n+1}\right), \quad n=2,3, \ldots
$$

These higher degree corrections to the modified energy $E_{I}^{2}$ may be of relevance in studying various qualitative aspects of the $\mathrm{KdV}$ evolution. However, for the purpose of showing GWP in $H^{s}(\mathbb{R})$ down to $s>-\frac{3}{4}$ and in $H^{s}(\mathbb{T})$ down to $s \geq-\frac{1}{2}$, we will see that almost conservation of $E_{I}^{4}(t)$ suffices.

The modified energy construction process is illustrated in the case of the Dirichlet energy

$$
E_{D}^{2}(t)=\left\|\partial_{x} u\right\|_{L_{x}^{2}}^{2}=\Lambda_{2}\left(\left(i \xi_{1}\right)\left(i \xi_{2}\right)\right) .
$$

Define $E_{D}^{3}(t)=E_{D}^{2}(t)+\Lambda_{3}\left(\sigma_{3}\right)$, and use (2.3) to see

$$
\partial_{t} E_{D}^{3}(t)=\Lambda_{3}\left(\left[i\left(\xi_{1}+\xi_{2}\right) i \xi_{3}\left\{\xi_{1}+\xi_{2}\right\}\right]_{s y m}\right)+\Lambda_{3}\left(\sigma_{3} \alpha_{3}\right)+\Lambda_{4}\left(M_{4}\right),
$$

where $M_{4}$ is explicitly obtained from $\sigma_{3}$. Noting that $i\left(\xi_{1}+\xi_{2}\right) i \xi_{3}\left\{\xi_{1}+\xi_{2}\right\}=-\xi_{3}^{3}$ on the set $\xi_{1}+\xi_{2}+\xi_{3}=0$, we know that

$$
\partial_{t} E_{D}^{3}(t)=\Lambda_{3}\left(-\frac{1}{3} \alpha_{3}\right)+\Lambda_{3}\left(\sigma_{3} \alpha_{3}\right)+\Lambda_{4}\left(M_{4}\right) .
$$

The choice of $\sigma_{3}=\frac{1}{3}$ results in a cancellation of the $\Lambda_{3}$ terms and

$$
M_{4}=\left[\left\{\xi_{1}+\xi_{2}\right\}\right]_{\text {sym }}=\xi_{1}+\xi_{2}+\xi_{3}+\xi_{4}
$$

so $M_{4}=0$.

Therefore, $E_{D}^{3}(t)=\Lambda_{2}\left(\left(i \xi_{1}\right)\left(i \xi_{2}\right)\right)+\Lambda_{3}\left(\frac{1}{3}\right)$ is an exactly conserved quantity. The modified energy construction applied to the Dirichlet energy led us to the Hamiltonian for KdV. Applying the construction to higher order derivatives in $L^{2}$ will similarly lead to the higher conservation laws of KdV. 


\section{Pointwise multiplier Bounds}

This section presents a detailed analysis of the multipliers $M_{3}, M_{4}, M_{5}$ which were introduced in the iteration process of the previous section. The analysis identifies cancellations resulting in pointwise upper bounds on these multipliers depending upon the relative sizes of the multiplier's arguments. These bounds are applied to prove an almost conservation property in the next section. We begin by recording some arithmetic and calculus facts.

4.1. Arithmetic and calculus facts. The following arithmetic facts may be easily verified:

$$
\xi_{1}+\xi_{2}+\xi_{3}+\xi_{4}=0 \Longrightarrow \alpha_{4}=\xi_{1}^{3}+\xi_{2}^{3}+\xi_{3}^{3}+\xi_{4}^{3}=3\left(\xi_{1}+\xi_{2}\right)\left(\xi_{1}+\xi_{3}\right)\left(\xi_{1}+\xi_{4}\right) .
$$

A related observation for the circle was exploited by C. Fefferman 19] and by Carleson and Sjölin [1] for curves with nonzero curvature. These properties were also observed by Rosales [47] and (4.1) was used by Bourgain in [5].

Definition 3. Let $a$ and $b$ be smooth functions of the real variable $\xi$. We say that $a$ is controlled by $b$ if $b$ is nonnegative and satisfies $b(\xi) \sim b\left(\xi^{\prime}\right)$ for $|\xi| \sim\left|\xi^{\prime}\right|$ and

$$
\begin{aligned}
a(\xi) & =O(b(\xi)), \\
a^{\prime}(\xi) & =O\left(\frac{b(\xi)}{|\xi|}\right), \\
a^{\prime \prime}(\xi) & =O\left(\frac{b(\xi)}{|\xi|^{2}}\right),
\end{aligned}
$$

for all nonzero $\xi$.

With this notion, we can state the following forms of the mean value theorem.

Lemma 4.1. If $a$ is controlled by $b$ and $|\eta| \ll|\xi|$, then

$$
a(\xi+\eta)-a(\xi)=O\left(|\eta| \frac{b(\xi)}{|\xi|}\right) .
$$

Lemma 4.2. If $a$ is controlled by $b$ and $|\eta|,|\lambda| \ll|\xi|$, then

$$
a(\xi+\eta+\lambda)-a(\xi+\eta)-a(\xi+\lambda)+a(\xi)=O\left(|\eta||\lambda| \frac{b(\xi)}{|\xi|^{2}}\right) .
$$

We will sometimes refer to our use of (4.4) as applying the double mean value theorem.

4.2. $M_{3}$ bound. The multiplier $M_{3}$ was defined in (3.3). In this section, we will generally be considering an arbitrary even $\mathbb{R}$-valued 1-multiplier $m$. We will specialize to the situation when $m$ is of the form (4.7) below. Recalling that $\xi_{1}+\xi_{2}+\xi_{3}=0$ and that $m$ is even allows us to re-express (3.3) as

$$
M_{3}\left(\xi_{1}, \xi_{2}, \xi_{3}\right)=-i\left[m^{2}\left(\xi_{1}\right) \xi_{1}\right]_{s y m}=-\frac{i}{3}\left[m^{2}\left(\xi_{1}\right) \xi_{1}+m^{2}\left(\xi_{2}\right) \xi_{2}+m^{2}\left(\xi_{3}\right) \xi_{3}\right] .
$$

Lemma 4.3. If $m$ is even $\mathbb{R}$-valued and $m^{2}$ is controlled by itself, then, on the set $\xi_{1}+\xi_{2}+\xi_{3}=0,\left|\xi_{i}\right| \sim N_{i}$ (dyadic),

$$
\left|M_{3}\left(\xi_{1}, \xi_{2}, \xi_{3}\right)\right| \lesssim \max \left(m^{2}\left(\xi_{1}\right), m^{2}\left(\xi_{2}\right), m^{2}\left(\xi_{3}\right)\right) \min \left(N_{1}, N_{2}, N_{3}\right) .
$$


Proof. Symmetry allows us to assume $N_{1}=N_{2} \geq N_{3}$. In case $N_{3} \ll N_{1}$, the claimed estimate is equivalent to showing

$$
m^{2}\left(\xi_{1}\right) \xi_{1}-m^{2}\left(\xi_{1}+\xi_{3}\right)\left(\xi_{1}+\xi_{3}\right)+m^{2}\left(\xi_{3}\right) \xi_{3} \leq \max \left(m^{2}\left(\xi_{1}\right), m^{2}\left(\xi_{3}\right)\right) N_{3} .
$$

But this easily follows when we rewrite the left side as $\left(m^{2}\left(\xi_{1}\right)-m^{2}\left(\xi_{1}+\xi_{3}\right)\right) \xi_{1}-$ $m^{2}\left(\xi_{1}+\xi_{3}\right) \xi_{3}+m^{2}\left(\xi_{3}\right) \xi_{3}$ and use (4.3). In case $N_{3} \sim N_{2}$, (4.6) may be directly verified.

In the particular case when the multiplier $m(\xi)$ is smooth, monotone, and of the form

$$
m(\xi)=\left\{\begin{array}{cc}
1, & |\xi|<N \\
N^{-s}|\xi|^{s}, & |\xi|>2 N
\end{array}\right.
$$

we have

$$
\left|M_{3}\left(\xi_{1}, \xi_{2}, \xi_{3}\right)\right| \leq \min \left(N_{1}, N_{2}, N_{3}\right)
$$

4.3. $M_{4}$ bound. This subsection establishes the following pointwise upper bound on the multiplier $M_{4}$.

Lemma 4.4. Assume $m$ is of the form (4.7). In the region where $\left|\xi_{i}\right| \sim N_{i}, \mid \xi_{j}+$ $\xi_{k} \mid \sim N_{j k}$ for $N_{i}, N_{j k}$ dyadic,

$$
\left|M_{4}\left(\xi_{1}, \xi_{2}, \xi_{3}, \xi_{4}\right)\right| \lesssim \frac{\left|\alpha_{4}\right| m^{2}\left(\min \left(N_{i}, N_{j k}\right)\right)}{\left(N+N_{1}\right)\left(N+N_{2}\right)\left(N+N_{3}\right)\left(N+N_{4}\right)} .
$$

We begin by deriving two explicit representations of $M_{4}$ in terms of $m$. These identities are then analyzed in cases to prove (4.9).

Recall that,

$$
M_{4}\left(\xi_{1}, \xi_{2}, \xi_{3}, \xi_{4}\right)=c\left[\sigma_{3}\left(\xi_{1}, \xi_{2}, \xi_{3}+\xi_{4}\right)\left(\xi_{3}+\xi_{4}\right)\right]_{s y m},
$$

where $\sigma_{3}=-\frac{M_{3}}{\alpha_{3}}$ and

$$
\begin{aligned}
M_{3}\left(x_{1}, x_{2}, x_{3}\right) & =-i\left[m\left(x_{1}\right) m\left(x_{2}+x_{3}\right)\left(x_{2}+x_{3}\right)\right]_{s y m} \\
& =-\frac{i}{3}\left[m^{2}\left(x_{1}\right) x_{1}+m^{2}\left(x_{2}\right) x_{2}+m^{2}\left(x_{3}\right) x_{3}\right],
\end{aligned}
$$

and $\alpha_{3}\left(x_{1}, x_{2}, x_{3}\right)=x_{1}^{3}+x_{2}^{3}+x_{3}^{3}=3 x_{1} x_{2} x_{3}$. We shall ignore the irrelevant constant in (4.10). Therefore,

$$
\begin{aligned}
M_{4}\left(\xi_{1}, \xi_{2}, \xi_{3}, \xi_{4}\right) & =-\frac{1}{2}\left[\frac{m^{2}\left(\xi_{1}\right) \xi_{1}+m^{2}\left(\xi_{2}\right) \xi_{2}+m^{2}\left(\xi_{3}+\xi_{4}\right)\left(\xi_{3}+\xi_{4}\right)}{3 \xi_{1} \xi_{2}}\right]_{s y m} \\
& =-\frac{1}{2}\left[\frac{2 m^{2}\left(\xi_{1}\right) \xi_{1}+m^{2}\left(\xi_{3}+\xi_{4}\right)\left(\xi_{3}+\xi_{4}\right)}{3 \xi_{1} \xi_{2}}\right]_{s y m} .
\end{aligned}
$$

Recall also from (4.2) that

$$
\begin{aligned}
\alpha_{4}\left(\xi_{1}, \xi_{2}, \xi_{3}, \xi_{4}\right) & =\xi_{1}^{3}+\xi_{2}^{3}+\xi_{3}^{3}+\xi_{4}^{3} \\
& =3\left(\xi_{1} \xi_{2} \xi_{3}+\xi_{1} \xi_{2} \xi_{4}+\xi_{1} \xi_{3} \xi_{4}+\xi_{2} \xi_{3} \xi_{4}\right) \\
& =3\left(\xi_{1}+\xi_{2}\right)\left(\xi_{1}+\xi_{3}\right)\left(\xi_{1}+\xi_{4}\right) .
\end{aligned}
$$


We can now rewrite the first term in (4.12)

$$
\begin{aligned}
{\left[\frac{2 m^{2}\left(\xi_{1}\right) \xi_{1} \xi_{3} \xi_{4}}{3 \xi_{1} \xi_{2} \xi_{3} \xi_{4}}\right]_{s y m}=} & \frac{2}{9}\left[\frac{m^{2}\left(\xi_{1}\right)\left(\xi_{1} \xi_{2} \xi_{3}+\xi_{1} \xi_{2} \xi_{4}+\xi_{1} \xi_{3} \xi_{4}+\xi_{2} \xi_{3} \xi_{4}-\xi_{2} \xi_{3} \xi_{4}\right)}{\xi_{1} \xi_{2} \xi_{3} \xi_{4}}\right]_{s y m} \\
= & \frac{1}{54}\left[m^{2}\left(\xi_{1}\right)+m^{2}\left(\xi_{2}\right)+m^{2}\left(\xi_{3}\right)+m^{2}\left(\xi_{4}\right)\right] \frac{\alpha_{4}}{\xi_{1} \xi_{2} \xi_{3} \xi_{4}} \\
& -\frac{1}{18}\left[\frac{m^{2}\left(\xi_{1}\right)}{\xi_{1}}+\frac{m^{2}\left(\xi_{2}\right)}{\xi_{2}}+\frac{m^{2}\left(\xi_{3}\right)}{\xi_{3}}+\frac{m^{2}\left(\xi_{4}\right)}{\xi_{4}}\right] .
\end{aligned}
$$

The second term in (4.12) is rewritten, using $\xi_{1}+\xi_{2}+\xi_{3}+\xi_{4}=0$, and the fact the $m$ is even,

$$
\begin{aligned}
& {\left[\frac{-m^{2}\left(\xi_{1}+\xi_{2}\right)\left(\xi_{1}+\xi_{2}\right) \xi_{3} \xi_{4}}{3 \xi_{1} \xi_{2} \xi_{3} \xi_{4}}\right]_{s y m} } \\
&=-\frac{1}{18}\left\{\frac{m^{2}\left(\xi_{1}+\xi_{2}\right)\left(\xi_{1} \xi_{3} \xi_{4}+\xi_{2} \xi_{3} \xi_{4}\right)+m^{2}\left(\xi_{3}+\xi_{4}\right)\left(\xi_{1} \xi_{2} \xi_{3}+\xi_{1} \xi_{3} \xi_{4}\right)}{\xi_{1} \xi_{2} \xi_{3} \xi_{4}}\right. \\
&+\frac{m^{2}\left(\xi_{1}+\xi_{3}\right)\left(\xi_{1} \xi_{2} \xi_{4}+\xi_{2} \xi_{3} \xi_{4}\right)+m^{2}\left(\xi_{2}+\xi_{4}\right)\left(\xi_{1} \xi_{2} \xi_{3}+\xi_{1} \xi_{3} \xi_{4}\right)}{\xi_{1} \xi_{2} \xi_{3} \xi_{4}} \\
&\left.\quad+\frac{m^{2}\left(\xi_{1}+\xi_{4}\right)\left(\xi_{1} \xi_{2} \xi_{3}+\xi_{2} \xi_{3} \xi_{4}\right)+m^{2}\left(\xi_{2}+\xi_{3}\right)\left(\xi_{1} \xi_{2} \xi_{4}+\xi_{1} \xi_{3} \xi_{4}\right)}{\xi_{1} \xi_{2} \xi_{3} \xi_{4}}\right\} \\
&=-\frac{1}{54} \frac{\alpha_{4}}{\xi_{1} \xi_{2} \xi_{3} \xi_{4}}\left[m^{2}\left(\xi_{1}+\xi_{2}\right)+m^{2}\left(\xi_{1}+\xi_{3}\right)+m^{2}\left(\xi_{1}+\xi_{4}\right)\right] .
\end{aligned}
$$

We record two identities for $M_{4}$.

Lemma 4.5. If $m$ is even and $\mathbb{R}$-valued, the following two identities for $M_{4}$ are valid:

$$
\begin{aligned}
M_{4}\left(\xi_{1}, \xi_{2}, \xi_{3}, \xi_{4}\right)= & -\frac{1}{108} \frac{\alpha_{4}}{\xi_{1} \xi_{2} \xi_{3} \xi_{4}}\left[m^{2}\left(\xi_{1}\right)+m^{2}\left(\xi_{2}\right)+m^{2}\left(\xi_{3}\right)+m^{2}\left(\xi_{4}\right)\right. \\
& \left.-m^{2}\left(\xi_{1}+\xi_{2}\right)-m^{2}\left(\xi_{1}+\xi_{3}\right)-m^{2}\left(\xi_{1}+\xi_{4}\right)\right] \\
& +\frac{1}{36}\left\{\frac{m^{2}\left(\xi_{1}\right)}{\xi_{1}}+\frac{m^{2}\left(\xi_{2}\right)}{\xi_{2}}+\frac{m^{2}\left(\xi_{3}\right)}{\xi_{3}}+\frac{m^{2}\left(\xi_{4}\right)}{\xi_{4}}\right\} \\
:= & I+I I .
\end{aligned}
$$

$$
\begin{aligned}
& M_{4}\left(\xi_{1}, \xi_{2}, \xi_{3}, \xi_{4}\right)=-\frac{1}{36} \frac{1}{\xi_{1} \xi_{2} \xi_{3} \xi_{4}} \times \\
& \left\{\xi_{1} \xi_{2} \xi_{3}\left[m^{2}\left(\xi_{1}\right)+m^{2}\left(\xi_{2}\right)+m^{2}\left(\xi_{3}\right)-m^{2}\left(\xi_{1}+\xi_{2}\right)-m^{2}\left(\xi_{1}+\xi_{3}\right)-m^{2}\left(\xi_{1}+\xi_{4}\right)\right]\right. \\
& +\xi_{1} \xi_{2} \xi_{4}\left[m^{2}\left(\xi_{1}\right)+m^{2}\left(\xi_{2}\right)+m^{2}\left(\xi_{4}\right)-m^{2}\left(\xi_{1}+\xi_{2}\right)-m^{2}\left(\xi_{1}+\xi_{3}\right)-m^{2}\left(\xi_{1}+\xi_{4}\right)\right] \\
& +\xi_{1} \xi_{3} \xi_{4}\left[m^{2}\left(\xi_{1}\right)+m^{2}\left(\xi_{3}\right)+m^{2}\left(\xi_{4}\right)-m^{2}\left(\xi_{1}+\xi_{2}\right)-m^{2}\left(\xi_{1}+\xi_{3}\right)-m^{2}\left(\xi_{1}+\xi_{4}\right)\right] \\
& \left.+\xi_{2} \xi_{3} \xi_{4}\left[m^{2}\left(\xi_{2}\right)+m^{2}\left(\xi_{3}\right)+m^{2}\left(\xi_{4}\right)-m^{2}\left(\xi_{1}+\xi_{2}\right)-m^{2}\left(\xi_{1}+\xi_{3}\right)-m^{2}\left(\xi_{1}+\xi_{4}\right)\right]\right\} .
\end{aligned}
$$

Proof. The identity (4.16) was established above. The identity (4.17) follows from (4.16) upon expanding $\alpha_{4}$ and writing the second term in (4.16) on a common denominator. 
Proof of Lemma 4.4 The proof consists of a case-by-case analysis pivoting on the relative sizes of $N_{i}, N_{j k}$. Symmetry properties of $M_{4}$ permit us to assume that $\left|\xi_{1}\right| \geq\left|\xi_{2}\right| \geq\left|\xi_{3}\right| \geq\left|\xi_{4}\right|$. Consequently, we assume $N_{1} \geq N_{2} \geq N_{3} \geq N_{4}$. Since $m^{2}(\xi)=1$ for $\xi<\frac{N}{2}$, a glance at (4.12) shows that $M_{4}$ vanishes when $\left|\xi_{1}\right|<\frac{N}{4}$. We may therefore assume that $\left|\xi_{1}\right| \gtrsim N$. Since $\xi_{1}+\xi_{2}+\xi_{3}+\xi_{4}=0$, we must also have $\left|\xi_{2}\right| \gtrsim N$.

From (4.13), we know that we can replace $\alpha_{4}$ on the right side of (4.9) by $N_{12} N_{13} N_{14}$. Suppose $N_{12}<\frac{N_{1}}{2}, N_{13}<\frac{N_{1}}{2}, N_{14}<\frac{N_{1}}{2}$. Then, $\xi_{1} \sim-\xi_{2}, \xi_{1} \sim-\xi_{3}$ and $\xi_{1} \sim-\xi_{4}$ so $\xi_{1}+\xi_{2}+\xi_{3}+\xi_{4} \sim-2 \xi_{1} \neq 0$. Thus, at least one of $N_{12}, N_{13}, N_{14}$ must be at least of size comparable to $N_{1}$. The right side of (4.9) may be reexpressed as

$$
\frac{N_{12} N_{13} N_{14} m^{2}\left(\min \left(N_{i}, N_{j k}\right)\right)}{N_{1}^{2}\left(N+N_{3}\right)\left(N+N_{4}\right)}
$$

Case 1. $\left|N_{4}\right| \gtrsim \frac{N}{2}$.

Term $I$ in (4.16) is bounded by $\frac{N_{12} N_{13} N_{14}}{N_{1}^{2} N_{3} N_{4}} m^{2}\left(\min \left(N_{i}, N_{j k}\right)\right)$, and therefore, after cancelling $\max \left(N_{12}, N_{13}, N_{14}\right)$ with one of the $N_{1}$, satisfies (4.9). Term $I I$ is treated next. In case $N_{12}, N_{13}, N_{14} \gtrsim N_{1}$, (4.18) is an upper bound of $\frac{N_{1}}{N_{3} N_{4}} m^{2}\left(N_{4}\right) \geq$ $\frac{m^{2}\left(N_{4}\right)}{N_{4}}$ and the triangle inequality gives $|I I| \lesssim \frac{m^{2}\left(N_{4}\right)}{N_{4}}$ since $\frac{m^{2}(\cdot)}{(\cdot)}$ is a decreasing function. If $N_{12} \gtrsim N_{1}, N_{13} \ll N_{1}$ and $N_{14} \gtrsim N_{1}$, we rewrite

$$
|I I| \sim\left\{\frac{m^{2}\left(\xi_{1}\right)}{\xi_{1}}+\frac{m^{2}\left(-\xi_{1}+\left(\xi_{1}+\xi_{3}\right)\right)}{\left(-\xi_{1}+\left(\xi_{1}+\xi_{3}\right)\right)}+\frac{m^{2}\left(\xi_{2}\right)}{\xi_{2}}+\frac{m^{2}\left(-\xi_{2}+\left(\xi_{2}+\xi_{4}\right)\right)}{\left(-\xi_{2}+\left(\xi_{2}+\xi_{4}\right)\right)}\right\} .
$$

Applying the mean value theorem and using $\xi_{1}+\xi_{2}+\xi_{3}+\xi_{4}=0$ gives $|I I| \lesssim$ $\left(\frac{m^{2}\left(\widetilde{\xi_{1}}\right)}{\xi_{1}}\right)^{\prime}\left(\xi_{1}+\xi_{3}\right) \lesssim \frac{N_{13}}{N_{1}^{2}} m^{2}\left(N_{1}\right)$ since $\widetilde{\xi_{1}}=\xi_{1}+O\left(N_{13}\right)$ and $N_{13} \ll N_{1}$, so this subcase is fine. If $N_{12} \ll N_{1}, N_{13} \ll N_{1}$ and $N_{14} \gtrsim N_{1}$, the double mean value theorem (4.4) applied to term $I I$ gives the bound

$$
|I I| \sim\left(\frac{m^{2}\left(\xi_{1}\right)}{\xi_{1}^{3}}\right)^{\prime \prime}\left(\xi_{1}+\xi_{2}\right)\left(\xi_{1}+\xi_{3}\right) .
$$

Our assumptions on $N_{12}, N_{13}$ give the bound $|I I| \lesssim \frac{N_{12} N_{13}}{N_{1}^{3}} m^{2}\left(N_{1}\right)$ which is smaller than (4.18).

The remaining subcases have either precisely one element of the set $\left\{N_{12}, N_{13}\right.$, $\left.N_{14}\right\}$ much smaller than $N_{1}$ or precisely two elements much smaller than $N_{1}$. In the case of just one small $N_{1 j}$, we apply the mean value theorem as above. When there are two small $N_{1 j}$, we apply the double mean value theorem as above.

Case 2. $\left|N_{4}\right| \ll \frac{N}{2}$.

Certainly, $m^{2}\left(\min \left(N_{i}, N_{j k}\right)\right)=1$ in this region. It is not possible for both $N_{12}<\frac{N_{1}}{4}$ and $N_{13}<\frac{N_{1}}{4}$ in this region. Indeed, we find then that $\xi_{1} \sim-\xi_{2}$ and $\xi_{1} \sim-\xi_{3}$ which with $\xi_{1}+\xi_{2}+\xi_{3}+\xi_{4}=0$ implies $\xi_{4} \sim \xi_{1}$ but $\left|\xi_{4}\right| \ll \frac{N}{2}$ while $\left|\xi_{1}\right| \sim N_{1} \gtrsim N$. We need to show $M_{4} \leq \frac{N_{12} N_{13}}{N_{1}\left(N+N_{3}\right) N}$.

Case 2A. $\frac{N_{1}}{4}>N_{12} \gtrsim \frac{N}{2}, N_{13} \sim N_{1}$.

Since $N_{4} \ll \frac{N}{2}$ and $\xi_{1}+\xi_{2}+\xi_{3}+\xi_{4}=0$, we must have $N_{12} \sim N_{3}$. So $N+N_{3} \sim N_{3}$ and our goal is to show $M_{4} \lesssim \frac{N_{12}}{N_{3} N} \sim \frac{1}{N}$. The last three terms in (4.17) are all $O\left(\frac{1}{N}\right)$, 
which is fine. The first term in (4.17) is

$$
\frac{1}{18 \xi_{4}}\left(m^{2}\left(\xi_{1}\right)+m^{2}\left(\xi_{2}\right)+m^{2}\left(\xi_{3}\right)-m^{2}\left(\xi_{1}+\xi_{2}\right)-m^{2}\left(\xi_{1}+\xi_{3}\right)-m^{2}\left(\xi_{1}+\xi_{4}\right)\right) .
$$

Replacing $\xi_{1}+\xi_{2}$ by $-\left(\xi_{3}+\xi_{4}\right)$ and $\xi_{1}+\xi_{3}$ by $-\left(\xi_{2}+\xi_{4}\right)$, we identify three differences poised for the mean value theorem. We find this term equals

$$
\frac{1}{18 \xi_{4}}\left[\left(m^{2}\left(\widetilde{\xi_{1}}\right)\right)^{\prime}+\left(m^{2}\left(\widetilde{\xi_{2}}\right)\right)^{\prime}+\left(m^{2}\left(\widetilde{\xi}_{3}\right)\right)^{\prime}\right] \xi_{4}
$$

with $\widetilde{\xi}_{i}=\xi_{i}+O\left(N_{4}\right)$ for $i=1,2,3$ so $\left|\widetilde{\xi}_{i}\right| \sim N_{i}$. This expression is also $O\left(\frac{1}{N}\right)$.

Case 2B. $N_{12} \ll \frac{N}{2}, N_{13} \sim N_{1}$.

Since $N_{12}=N_{34}$ and $N_{4} \ll \frac{N}{2}$, we must have $N_{3} \ll \frac{N}{2}$. We have $N_{13} \sim N_{1}$ and $N_{14} \sim N_{1}$ here so our desired upper bound is $\frac{N_{12}}{N^{2}}$. We recall (4.16) and evaluate $m^{2}$ when we can to find

$$
\begin{aligned}
M_{4}\left(\xi_{1}, \xi_{2}, \xi_{3}, \xi_{4}\right)= & \frac{\alpha_{4}}{54 \xi_{1} \xi_{2} \xi_{3} \xi_{4}}\left(m^{2}\left(\xi_{1}\right)+m^{2}\left(\xi_{2}\right)+2-1-m^{2}\left(\xi_{1}+\xi_{3}\right)-m^{2}\left(\xi_{1}+\xi_{4}\right)\right) \\
& -\frac{1}{18}\left(\frac{m^{2}\left(\xi_{1}\right)}{\xi_{1}}+\frac{m^{2}\left(\xi_{2}\right)}{\xi_{2}}+\frac{\xi_{3}+\xi_{4}}{\xi_{3} \xi_{4}}\right) .
\end{aligned}
$$

The last term is dangerous so we isolate a piece of the first term to cancel it out. Expanding $\alpha_{4}=3\left(\xi_{1}+\xi_{2}\right)\left(\xi_{1}+\xi_{3}\right)\left(\xi_{1}+\xi_{4}\right)$, we see that

$$
\begin{aligned}
\frac{\alpha_{4}}{54 \xi_{1} \xi_{2} \xi_{3} \xi_{4}} & =\frac{1}{18} \frac{\left(\xi_{3}+\xi_{4}\right)}{\xi_{3} \xi_{4}} \frac{\left(\xi_{2}+\xi_{4}\right)\left(\xi_{1}+\xi_{4}\right)}{\xi_{1} \xi_{2}} \\
& =\frac{1}{18} \frac{\left(\xi_{3}+\xi_{4}\right)}{\xi_{3} \xi_{4}}\left(1+\frac{\xi_{4}\left(\xi_{1}+\xi_{2}+\xi_{4}\right)}{\xi_{1} \xi_{2}}\right) \\
& =\frac{1}{18} \frac{\left(\xi_{3}+\xi_{4}\right)}{\xi_{3} \xi_{4}}\left(1-\frac{\xi_{4} \xi_{3}}{\xi_{1} \xi_{2}}\right) .
\end{aligned}
$$

The first piece cancels with $-\frac{1}{18} \frac{\xi_{3}+\xi_{4}}{\xi_{3} \xi_{4}}$ in (4.19) and the second piece is of size $\frac{N_{12}}{N_{1}^{2}}$, which is fine. It remains to control

$$
\frac{\alpha_{4}}{54 \xi_{1} \xi_{2} \xi_{3} \xi_{4}}\left(m^{2}\left(\xi_{1}\right)+m^{2}\left(\xi_{2}\right)-m^{2}\left(\xi_{1}+\xi_{3}\right)-m^{2}\left(\xi_{1}+\xi_{4}\right)\right)-\frac{1}{18}\left(\frac{m^{2}\left(\xi_{1}\right)}{\xi_{1}}+\frac{m^{2}\left(\xi_{2}\right)}{\xi_{2}}\right),
$$

by $\frac{N_{12}}{N^{2}}$. Expand $\alpha_{4}$ using (4.13) to rewrite this expression as

$$
\begin{aligned}
& \frac{3\left(\xi_{1} \xi_{2} \xi_{3}+\xi_{1} \xi_{2} \xi_{4}\right)}{54 \xi_{1} \xi_{2} \xi_{3} \xi_{4}}\left(m^{2}\left(\xi_{1}\right)+m^{2}\left(\xi_{2}\right)-m^{2}\left(\xi_{1}+\xi_{3}\right)-m^{2}\left(\xi_{1}+\xi_{4}\right)\right) \\
& -\frac{3\left(\xi_{1} \xi_{3} \xi_{4}+\xi_{2} \xi_{3} \xi_{4}\right)}{54 \xi_{1} \xi_{2} \xi_{3} \xi_{4}}\left(m^{2}\left(\xi_{1}+\xi_{3}\right)+m^{2}\left(\xi_{1}+\xi_{4}\right)\right) \\
& +\frac{1}{18 \xi_{1} \xi_{2}}\left[\xi_{1} m^{2}\left(\xi_{1}\right)+\xi_{2} m^{2}\left(\xi_{2}\right)\right] .
\end{aligned}
$$

(The second term in (4.20) cancelled with part of the first.) The second and third terms in (4.21) are $O\left(\frac{N_{12}}{N^{2}}\right)$ and may therefore be ignored. We rewrite the first term in (4.21) using the fact that $m^{2}$ is even as

$$
\frac{3\left(\xi_{1} \xi_{2} \xi_{3}+\xi_{1} \xi_{2} \xi_{4}\right)}{54 \xi_{1} \xi_{2} \xi_{3} \xi_{4}}\left(m^{2}\left(-\xi_{1}\right)+m^{2}\left(\xi_{2}\right)-m^{2}\left(-\left(\xi_{1}+\xi_{3}\right)\right)-m^{2}\left(-\left(\xi_{1}+\xi_{4}\right)\right)\right) .
$$


Since $-\xi_{1}+\xi_{2}+\left(\xi_{1}+\xi_{3}\right)+\left(\xi_{1}+\xi_{4}\right)=0$, we can apply the double mean value theorem to obtain

$$
=\frac{3\left(\xi_{1} \xi_{2} \xi_{3}+\xi_{1} \xi_{2} \xi_{4}\right)}{54 \xi_{1} \xi_{2} \xi_{3} \xi_{4}}\left(m^{2}\left(-\widetilde{\xi}_{1}\right)\right)^{\prime \prime} \xi_{3} \xi_{4}
$$

with $-\widetilde{\xi}_{1}=-\xi_{1}+O\left(N_{3}\right)+O\left(N_{4}\right) \Longrightarrow\left|-\widetilde{\xi}_{1}\right| \sim N_{1}$. Therefore, this term is bounded by

$$
\frac{\xi_{1} \xi_{2} \xi_{3}+\xi_{1} \xi_{2} \xi_{4}}{\xi_{1} \xi_{2} \xi_{3} \xi_{4}} \frac{m^{2}\left(-\widetilde{\xi}_{1}\right)}{\left(-\widetilde{\xi}_{1}\right)^{2}} \xi_{3} \xi_{4}=O\left(\frac{N_{12}}{N_{1}^{2}} m^{2}\left(\widetilde{\xi_{1}}\right)\right)
$$

which is smaller than $\frac{N_{12}}{N_{1}^{2}}$ as claimed.

Case 2C. $\frac{N_{1}}{4}>N_{13} \gtrsim \frac{N}{2}, N_{12} \sim N_{1}$.

This case follows from a modification of Case $2 \mathrm{~A}$.

Case 2D. $N_{13} \ll \frac{N}{2}, N_{12} \sim N_{1}$.

This case does not occur because $N_{13} \sim N_{24}$ but $N_{4}$ is very small which forces $N_{2}$ to also be small, which is a contradiction.

4.4. $M_{5}$ bound. The multiplier $M_{5}$ was defined in (3.9), with $\sigma_{4}=-\frac{M_{4}}{\alpha_{4}}$. Our work on $M_{4}$ above showed that $M_{4}$ vanishes whenever $\alpha_{4}$ vanishes so there is no denominator singularity in $M_{5}$. Moreover, we have the following upper bound on $M_{5}$ in the particular case when $m$ is of the form (4.7).

Lemma 4.6. If $m$ is of the form (4.7), then

$$
\left|M_{5}\left(\xi_{1}, \ldots, \xi_{5}\right)\right| \lesssim\left[\frac{m^{2}\left(N_{* 45}\right) N_{45}}{\left(N+N_{1}\right)\left(N+N_{2}\right)\left(N+N_{3}\right)\left(N+N_{45}\right)}\right]_{s y m},
$$

where

$$
N_{* 45}=\min \left(N_{1}, N_{2}, N_{3}, N_{45}, N_{12}, N_{13}, N_{23}\right) .
$$

Proof. This follows directly from Lemma 4.4. Note that $\xi_{1}+\xi_{4}+\xi_{5}=-\left(\xi_{2}+\xi_{3}\right)$ allows for the simplification in defining $N_{* 45}$.

\section{Quintilinear estimate on $\mathbb{R}$}

The $M_{5}$ upper bound contained in Lemma 4.6 and the local well-posedness machinery [31, [5], 32] are applied to prove an almost conservation property of the modified energy $E_{I}^{4}$. The almost conservation of $E_{I}^{4}$ is the key ingredient in our proof of global well-posedness of the initial value problem for $\mathrm{KdV}$ with rough initial data.

Recall that $X_{s, b}^{\delta}$ denotes the Bourgain space [5] associated to the cubic $\left\{\tau=\xi^{3}\right\}$ on the time interval $[0, \delta]$. We begin with a quintilinear estimate.

Lemma 5.1. Let $w_{i}(x, t)$ be functions of space-time. Then

$$
\left|\int_{0}^{\delta} \int \prod_{i=1}^{5} w_{i}(x, t) d x d t\right| \lesssim\left(\prod_{j=1}^{3}\left\|w_{j}\right\|_{X_{\frac{1}{4}, \frac{1}{2}+}^{\delta}}\right)\left\|w_{4}\right\|_{X_{-\frac{3}{4}, \frac{1}{2}+}^{\delta}}\left\|w_{5}\right\|_{X_{-\frac{3}{4}, \frac{1}{2}+}^{\delta}} .
$$

Proof. The left side of (5.1) is estimated via Hölder's inequality by

$$
\left(\prod_{j=1}^{3}\left\|w_{j}\right\|_{L_{x}^{4} L_{t \in[0, \delta]}^{\infty}}\right)\left\|w_{4}\right\|_{L_{x}^{8} L_{t \in[0, \delta]}^{2}}\left\|w_{5}\right\|_{L_{x}^{8} L_{t \in[0, \delta]}^{2}} .
$$


The first three factors are bounded using a maximal inequality from [31,

$$
\|w\|_{L_{x}^{4} L_{t \in[0, \delta]}^{\infty}} \lesssim\|w\|_{X_{\frac{1}{4}, \frac{1}{2}+}^{\delta}} .
$$

(Strictly speaking, 31] contains an estimate for $S(t) \phi$ which implies (5.2) by summing over cubic levels using $b=\frac{1}{2}+$; see [5] or [22, [24]. A similar comment applies to (5.5) below.) The $w_{4}, w_{5}$ terms are controlled using the smoothing estimate

$$
\|w\|_{L_{x}^{8} L_{t}^{2}} \leq\|w\|_{X_{-\frac{3}{4}, \frac{1}{2}+}}
$$

which is an interpolant between the local-in-time energy estimate

$$
\|w\|_{L_{x}^{2} L_{t}^{2} \in[0, \delta]} \lesssim\|u\|_{X_{0, \frac{1}{2}+}^{\delta}}
$$

and the Kato smoothing estimate [31], valid for $\widehat{w}$ supported outside $\{|\xi|<1\}$,

$$
\|w\|_{L_{x}^{\infty} L_{t \in[0, \delta]}^{2}} \lesssim\|w\|_{X_{-1, \frac{1}{2}+}^{\delta}} .
$$

In the remaining low frequency cases (e.g., when $\widehat{w_{4}}$ is supported inside $[-1,1]$ ) we have $\left\|w_{4}\right\|_{L_{x}^{\infty} L_{t \in[0, \delta]}^{\infty}} \leq\left\|w_{4}\right\|_{X_{0, \frac{1}{2}+}^{\delta}}$ and therefore we may easily control $\left\|w_{4}\right\|_{L_{x}^{8} L_{t \in[0, \delta]}^{2}}$ by $\|w\|_{X_{-\frac{3}{4}, \frac{1}{2}+}^{\delta}}$.

Lemma 5.1 is combined with the $M_{5}$ upper bound of Lemma 4.6 in the next result.

Lemma 5.2. Recall the definition (3.1) of the operator I. If the associated multiplier $m$ is of the form (4.7) with $s=-\frac{3}{4}+$, then

$$
\left|\int_{0}^{\delta} \Lambda_{5}\left(M_{5} ; u_{1}, \ldots, u_{5}\right) d t\right| \lesssim N^{-\beta} \prod_{j=1}^{5}\left\|I u_{j}\right\|_{X_{0, \frac{1}{2}+}^{\delta}},
$$

with $\beta=3+\frac{3}{4}-$.

Proof. We may assume that the functions $\widehat{u_{j}}$ are nonnegative. By a LittlewoodPaley decomposition, we restrict each $\widehat{u_{j}}$ to a frequency band $\left|\xi_{j}\right| \sim N_{j}$ (dyadic) and sum in the $N_{j}$ at the end of the argument. The definition of the operator $I$ and (4.22) shows that it suffices to prove

$$
\begin{aligned}
\mid \int_{0}^{\delta} & \Lambda_{5}\left(\frac{N_{45} m^{2}\left(N_{* 45}\right)}{\left(N+N_{1}\right)\left(N+N_{2}\right)\left(N+N_{3}\right)\left(N+N_{45}\right) m\left(N_{1}\right) \cdots m\left(N_{5}\right)} ; u_{1}, \ldots, u_{5}\right) d t \mid \\
& \lesssim N^{-\beta} \prod_{j=1}^{5} N_{j}^{0-}\left\|u_{j}\right\|_{X_{0, \frac{1}{2}+}^{\delta}} .
\end{aligned}
$$

We cancel $\frac{N_{45}}{N+N_{45}} \leq 1$ and consider the worst case when $m^{2}\left(N_{* 45}\right)=1$ throughout.

Note that $M_{4}$ vanishes when $\left|\xi_{i}\right| \ll N$ for $i=1,2,3,4$. Hence, we are allowed to assume at least one, and hence two, of the $N_{i} \gtrsim N$. Symmetry allows us to assume $N_{1} \geq N_{2} \geq N_{3}$ and $N_{4} \geq N_{5}$. 
The objective here is to show that

$$
\begin{aligned}
& \left|\int_{0}^{\delta} \Lambda_{5}\left(\prod_{i=1}^{3} \frac{1}{\left(N+N_{i}\right) m\left(N_{i}\right)} \frac{1}{m\left(N_{4}\right)} \frac{1}{m\left(N_{5}\right)} ; u_{1}, \ldots, u_{5}\right) d t\right| \\
& \quad \lesssim N^{-\frac{15}{4}+} \prod_{j=1}^{5} N_{j}^{0-}\left\|u_{j}\right\|_{X_{0, \frac{1}{2}+}^{\delta}} .
\end{aligned}
$$

The form (4.7) of $m$ with $s=-\frac{3}{4}+$ implies that $\frac{1}{\left(N+N_{i}\right) m\left(N_{i}\right)} \lesssim N^{-\frac{3}{4}+}\left\langle N_{i}\right\rangle^{-\frac{1}{4}-}$. Therefore, we need to control

$$
N^{-\frac{9}{4}+} \int_{0}^{\delta} \Lambda_{5}\left(\left\langle N_{1}\right\rangle^{-\frac{1}{4}-}\left\langle N_{2}\right\rangle^{-\frac{1}{4}-}\left\langle N_{3}\right\rangle^{-\frac{1}{4}-} \frac{1}{m\left(N_{4}\right)} \frac{1}{m\left(N_{5}\right)}\right) d t .
$$

We break the analysis into three main cases: Case 1. $N_{4}, N_{5} \gtrsim N$; Case 2. $N_{4} \gtrsim N \gg N_{5}$; Case 3. $N \gg N_{4} \geq N_{5}$.

In Case 1, we have that $\frac{1}{m\left(N_{4}\right)} \sim N^{-\frac{3}{4}+}\left\langle N_{4}\right\rangle^{\frac{3}{4}-}$ and $\frac{1}{m\left(N_{5}\right)} \sim N^{-\frac{3}{4}+}\left\langle N_{5}\right\rangle^{\frac{3}{4}-}$. The desired prefactor $N^{-\frac{15}{4}+}$ then appears and (5.1) gives the result claimed.

In Case 2, $m\left(N_{5}\right)=1$ and we must have $N_{1} \geq N \geq N_{5}$ so we multiply by $\left(\frac{N_{1}}{N}\right)^{\frac{3}{4}}\left(\frac{N_{1}}{N_{5}}\right)^{\frac{1}{4}} \geq 1$ and it suffices to bound

$$
N^{-\frac{3}{4}} N^{-\frac{12}{4}+}\left|\int_{0}^{\delta} \Lambda_{5}\left(\left\langle N_{1}\right\rangle^{\frac{3}{4}}\left\langle N_{2}\right\rangle^{-\frac{1}{4}-}\left\langle N_{3}\right\rangle^{-\frac{1}{4}-}\left\langle N_{4}\right\rangle^{\frac{3}{4}}\left\langle N_{5}\right\rangle^{-\frac{1}{4}-}\right) d t\right|
$$

which may be done using (5.1).

For Case 3 , we have $m\left(N_{4}\right)=m\left(N_{5}\right)=1$. We are certain to have $N_{1} \geq N_{2} \geq N$ and can therefore multiply by

$$
\frac{N_{1}}{N^{\frac{3}{4}}\left\langle N_{4}\right\rangle^{\frac{1}{4}}} \frac{N_{2}}{N^{\frac{3}{4}}\left\langle N_{5}\right\rangle^{\frac{1}{4}}} \geq 1
$$

to again encounter (5.7).

A glance back at (3.8) shows that for solutions of $K d V$, we can now control the increment of the modified energy $E_{I}^{4}$.

\section{Global Well-Posedness of KdV on $\mathbb{R}$}

The goal of this section is to construct the solution of the initial value problem (1.1) on an arbitrary fixed time interval $[0, T]$. We first state a variant of the local well-posedness result of [32]. Next, we perform a rescaling under which the variant local result has an existence interval of size 1 and the initial data is small. This rescaling is possible because the scaling invariant Sobolev index for $K d V$ is $-\frac{3}{2}$ which is much less than $-\frac{3}{4}$. Under the rescaling, we show that (3.8) and (5.6) allow us to iterate the local result many times with an existence interval of size 1 , thereby extending the local-in-time result to a global one. This will prove Theorem 1. 
6.1. A variant local well-posedness result. The expression $\|I u(t)\|_{L^{2}}$, where $\widehat{I u(t)}(\xi)=m(\xi) \widehat{u(t)}(\xi)$ and $m$ is of the form (4.7), is closely related to the $H^{s}(\mathbb{R})$ norm of $u$. Recall that the definition of $m$ in (4.7) depends upon $s$. An adaptation of the local well-posedness result in [32], along the lines of Lemma 5.2 in [16] and Section 12 in [17], establishes the following result.

Proposition 2. If $s>-\frac{3}{4}$, the initial value problem (1.1) is locally well-posed for data $\phi$ satisfying $I \phi \in L^{2}(\mathbb{R})$. Moreover, the solution exists on a time interval $[0, \delta]$ with the lifetime

$$
\delta \sim\|I \phi\|_{L^{2}}^{-\alpha}, \alpha>0
$$

and the solution satisfies the estimate

$$
\|I u\|_{X_{0, \frac{1}{2}+}^{\delta}} \lesssim\|I \phi\|_{L^{2}}
$$

We briefly describe why this result follows from the arguments in 32 . The norm $\|I u\|_{L^{2}}$ is connected to the norm $\|u\|_{H^{s}}$ by the identity $\|I u\|_{L^{2}}=\|D u\|_{H^{s}}$ where $D$ is the Fourier multiplier operator with symbol

$$
d(\xi)=\frac{m(\xi)}{\langle\xi\rangle^{s}}= \begin{cases}\langle\xi\rangle^{-s}, & |\xi| \lesssim N \\ N^{-s}, & |\xi| \gg N\end{cases}
$$

Since $-\frac{3}{4}<s<0, d$ is essentially nondecreasing and $d(\xi) \gtrsim 1$ so $D$ acts like a differential operator. The crucial bilinear estimate required to prove Proposition 2 is

which is equivalent to showing

$$
\left\|I(u v)_{x}\right\|_{X_{0,-\frac{1}{2}+}} \lesssim\|I u\|_{X_{0, \frac{1}{2}+}}\|I v\|_{X_{0, \frac{1}{2}+}},
$$

$$
\left\|D(u v)_{x}\right\|_{X_{s,-\frac{1}{2}+}} \lesssim\|D u\|_{X_{s, \frac{1}{2}+}}\|D v\|_{X_{s, \frac{1}{2}+}} .
$$

Since $d\left(\xi_{1}+\xi_{2}\right) \lesssim d\left(\xi_{1}\right)+d\left(\xi_{2}\right)$, the operator $D$ may be moved onto the higher frequency factor inside the parenthesis in the left side of (6.3) and the bilinear estimate of [32] then proves (6.3).

6.2. Rescaling. Our goal is to construct the solution of (1.1) on an arbitrary fixed time interval $[0, T]$. We rescale the solution by writing $u_{\lambda}(x, t)=\lambda^{-2} u\left(\frac{x}{\lambda}, \frac{t}{\lambda^{3}}\right)$. We achieve the goal if we construct $u_{\lambda}$ on the time interval $\left[0, \lambda^{3} T\right]$. A calculation shows that

$$
\left\|I \phi_{\lambda}\right\|_{L^{2}} \lesssim \lambda^{-\frac{3}{2}-s} N^{-s}\|\phi\|_{H^{s}} .
$$

The choice of the parameter $N=N(T)$ will be made later but we select $\lambda$ now by requiring

$$
\lambda^{-\frac{3}{2}-s} N^{-s}\|\phi\|_{H^{s}}=\epsilon_{0}<1 \Longrightarrow \lambda \sim N^{-\frac{2 s}{3+2 s}} .
$$

We drop the $\lambda$ subscript on $u$ so that

$$
\|I \phi\|_{L^{2}}=\epsilon_{0}<1
$$

and the task is to construct the solution of (1.1) on the time interval $\left[0, \lambda^{3} T\right]$.

Remark 6.1. The spatial domain for the initial value problem (1.1) is $\mathbb{R}$ which is invariant under the rescaling $x \longmapsto \frac{x}{\lambda}$. In contrast, the spatial domain $\mathbb{T}$ for the periodic initial value problem for $K d V$ scales with $\lambda$. The adaptation of our proof of global well-posedness in the periodic context presented in Section 8 requires us to identify the dependence of various estimates on the spatial period. 
6.3. Almost conservation. Recall the modified energy $E_{I}^{2}(0)=\|I \phi\|_{L^{2}}^{2}$ $=\Lambda_{2}\left(m\left(\xi_{1}\right) m\left(\xi_{2}\right)\right)(0)$. This subsection shows that the modified energy $E_{I}^{2}(t)$ of our rescaled local-in-time solution $u$ is comparable to the modified energy $E_{I}^{4}(t)$. Next, as forecasted in Section 5, we use (3.8) and the bound (5.6) to show $E_{I}^{4}(t)$ is almost conserved, implying almost conservation of $E_{I}^{2}(t)=\|I u(t)\|_{L^{2}}^{2}$. Since the lifetime of the local result (6.1) is controlled by $\|I \phi\|_{L^{2}}^{2}$, this conservation property permits us to iterate the local result with the same sized existence interval.

Lemma 6.1. Let $I$ be defined with the multiplier $m$ of the form (4.7) and $s=-\frac{3}{4}+$. Then

$$
\left|E_{I}^{4}(t)-E_{I}^{2}(t)\right| \lesssim\|I u(t)\|_{L^{2}}^{3}+\|I u(t)\|_{L^{2}}^{4} .
$$

Remark 6.2. The estimate 6.6 is an a priori estimate for functions of $x$ alone. The variable $t$ appears as a parameter.

Proof. Since $E_{I}^{4}(t)=E_{I}^{2}(t)+\Lambda_{3}\left(\sigma_{3}\right)+\Lambda_{4}\left(\sigma_{4}\right)$, it suffices to prove

$$
\begin{gathered}
\left|\Lambda_{3}\left(\sigma_{3} ; u_{1}, u_{2}, u_{3}\right)\right| \lesssim \prod_{j=1}^{3}\left\|I u_{j}(t)\right\|_{L^{2}}, \\
\left|\Lambda_{4}\left(\sigma_{4} ; u_{1}, \ldots, u_{4}\right)\right| \lesssim \prod_{j=1}^{4}\left\|I u_{j}(t)\right\|_{L^{2}} .
\end{gathered}
$$

We may again assume that the $\widehat{u_{j}}$ are nonnegative. By the definitions of $\sigma_{3}$ (3.5), and $I$ (3.1), and also (4.1) and (4.5), (6.7) follows if we show

$$
\left|\Lambda_{3}\left(\frac{m^{2}\left(\xi_{1}\right) \xi_{1}+m^{2}\left(\xi_{2}\right) \xi_{2}+m^{2}\left(\xi_{3}\right) \xi_{3}}{\xi_{1} \xi_{2} \xi_{3} m\left(\xi_{1}\right) m\left(\xi_{2}\right) m\left(\xi_{3}\right)} ; u_{1}, u_{2}, u_{3}\right)\right| \lesssim \prod_{j=1}^{3}\left\|u_{j}\right\|_{2} .
$$

We make a Littlewood-Paley decomposition and restrict attention to the contribution arising from $\left|\xi_{i}\right| \sim N_{i}$ (dyadic), and without loss assume $N_{1} \geq N_{2} \geq N_{3}$. In case $N_{1}<\frac{1}{2} N$, then $m^{2}\left(\xi_{i}\right)=1, i=1,2,3 \Longrightarrow \Lambda_{3}=0$. So, we can assume $N_{1} \sim N_{2} \geq N_{3}$. We consider separately the cases: $N_{3} \ll N, N_{3} \gtrsim N$.

I. $N_{3} \ll N$.

Since $\xi_{1}+\xi_{2}+\xi_{3}=0$ and $m^{2}$ controls itself (recall Lemma 4.1), we may apply (4.3) to show $\left|m^{2}\left(\xi_{1}\right) \xi_{1}+m^{2}\left(\xi_{2}\right) \xi_{2}+m^{2}\left(\xi_{3}\right) \xi_{3}\right| \sim N_{3}$. Of course $m\left(N_{3}\right)=1$ in this case so we need to bound $\Lambda_{3}\left(\frac{N^{s}}{N_{1}^{1+s}} \frac{N^{s}}{N_{1}^{1+s}}\right)$. But this quantity is bounded by $\Lambda_{3}\left(N_{1}^{-\frac{1}{6}} N_{2}^{-\frac{1}{6}} N_{3}^{-\frac{1}{6}}\right.$ ) (in fact with a decay in $N$ ) and we wish to prove

$$
\int_{\xi_{1}+\xi_{2}+\xi_{3}=0,\left|\xi_{i}\right| \sim N_{i}} \prod_{i=1}^{3} N_{i}^{-\frac{1}{6}} \widehat{u}_{i}\left(\xi_{i}\right) \lesssim \prod_{i=1}^{3}\left\|u_{i}\right\|_{L^{2}}
$$

Let $w_{i}(x)$ be defined via

$$
\widehat{w_{i}}(\xi)=N_{i}^{\frac{1}{6}} \widehat{u}_{i}(\xi) \chi_{\left\{|\xi| \sim N_{i}\right\}}(\xi) .
$$


The left side of (6.10) may be rewritten

$$
\begin{gathered}
\int_{\xi_{3}} \widehat{w_{3}}\left(-\xi_{3}\right) \int_{\xi_{3}=\xi_{1}+\xi_{2}} \widehat{w_{1}}\left(\xi_{1}\right) \widehat{w_{2}}\left(\xi_{2}\right)=\left\langle\widehat{w_{3}}, \widehat{u_{1} u_{2}}\right\rangle \\
=\left\langle\overline{w_{3}}, w_{1} w_{2}\right\rangle=\int w_{3} w_{1} w_{2} d x .
\end{gathered}
$$

We may now apply Hölder in $L_{x}^{3} L_{x}^{3} L_{x}^{3}$ to bound the left side of (6.10) by

$$
\left\|v_{3}\right\|_{L_{x}^{3}}\left\|w_{1}\right\|_{L_{x}^{3}}\left\|w_{2}\right\|_{L_{x}^{3}} \text {. }
$$

Finally, the form of $w_{i}$ (and hence $v_{3}$ ) given in (6.11) allows us to conclude using Sobolev that

$$
\left\|w_{i}\right\|_{L_{x}^{3}} \lesssim\left\|u_{i}\right\|_{L_{x}^{2}} .
$$

Remark 6.3. The argument reducing the left side of (6.10 to 6.13) by passing through the convolution representation (6.12) will appear many times below. We will often compress this discussion by referring to it as an " $L_{x}^{3} L_{x}^{3} L_{x}^{3}$ Hölder application".

II. $N_{3} \geq N$.

By definition of $m$, we have

$$
\left|m^{2}\left(\xi_{1}\right) \xi_{1}+m^{2}\left(\xi_{2}\right) \xi_{2}+m^{2}\left(\xi_{3}\right) \xi_{3}\right| \lesssim N^{-2 s}\left(N_{1}^{1+2 s}+N_{2}^{1+2 s}+N_{3}^{1+2 s}\right) .
$$

Suppose $s=-\frac{3}{4}+<-\frac{1}{2}$. Then this expression is

$$
\sim N^{-2 s} N_{3}^{1+2 s} \text {. }
$$

Therefore, the multiplier in (6.9) is bounded by

$$
\begin{aligned}
\frac{N_{3}^{1+2 s} N^{-2 s} N^{3 s}}{N_{1}^{1+s} N_{2}^{1+s} N_{3}^{1+s}} & \lesssim \frac{N_{3}^{s} N^{s}}{N_{1}^{1+s} N_{2}^{1+s}} \sim N^{s} \frac{N_{3}^{s+\frac{1}{6}}}{N_{1}^{1+s} N_{2}^{1+s} N_{3}^{\frac{1}{6}}} \\
& \lesssim N^{-\frac{3}{2}+\frac{1}{6}} N_{1}^{-\frac{1}{6}} N_{2}^{-\frac{1}{6}} N_{3}^{-\frac{1}{6}}
\end{aligned}
$$

and $L^{3} L^{3} L^{3}$ Hölder finishes off (6.9) and establishes (6.7).

We record here that the preceding calculations imply

$$
\left|\frac{m^{2}\left(\xi_{1}\right) \xi_{1}+m^{2}\left(\xi_{2}\right) \xi_{2}+m^{2}\left(\xi_{3}\right) \xi_{3}}{\xi_{1} \xi_{2} \xi_{3} m\left(\xi_{1}\right) m\left(\xi_{2}\right) m\left(\xi_{3}\right)}\right| \lesssim N^{-\frac{5}{4}+} N_{1}^{-\frac{1}{4}+} N_{2}^{-\frac{1}{4}+} N_{3}^{-\frac{1}{4}+} .
$$

We turn our attention to proving (6.8). By (4.9) (4.2), and the definition of $\sigma_{4}$ (3.7), it suffices to control for $|\xi| \sim N_{i}$ (dyadic), with $N_{1} \geq N_{2} \geq N_{3} \geq N_{4} \Longrightarrow$ $N_{1}=N_{2}$, that

$$
\begin{aligned}
\Lambda_{4} & \left(\frac{1}{\left(N+N_{1}\right)\left(N+N_{2}\right)\left(N+N_{3}\right)\left(N+N_{4}\right) m\left(N_{1}\right) m\left(N_{2}\right) m\left(N_{3}\right) m\left(N_{4}\right)} ; u_{1}, u_{2}, u_{3}, u_{4}\right) \\
& \lesssim \prod_{j=1}^{4}\left\|u_{j}\right\|_{L^{2}} .
\end{aligned}
$$

The definition of $m$ shows the multiplier appearing in the left side of (6.15) is

$$
\lesssim \frac{N^{4 s}}{N_{1}^{1+s} N_{2}^{1+s} N_{3}^{1+s} N_{4}^{1+s}}
$$


and for $s=-\frac{3}{4}+$,

$$
\lesssim N^{4 s} \frac{1}{N_{3}^{\frac{1}{2}+} N_{4}^{\frac{1}{2}+}}
$$

With this upper bound on the multiplier, we bound the left side of 6.15) in $L^{2} L^{2} L^{\infty} L^{\infty}$ via Hölder and Sobolev to obtain the estimate (6.15) and therefore (6.8).

Since our rescaled solution satisfies $\|I \phi\|_{L^{2}}^{2}=\epsilon_{0}^{2}<1$, we are certain that

$$
E_{I}^{4}(0)=E_{I}^{2}(0)+O\left(\epsilon_{0}^{3}\right)
$$

and, moreover, that

$$
E_{I}^{4}(t)=E_{I}^{2}(t)+O\left(\epsilon_{0}^{3}\right)
$$

whenever $\|I u(t)\|_{L^{2}}^{2}=E_{I}^{2}(t)<2 \epsilon_{0}$. Using the estimate (5.6) in (3.8), the rescaled solution is seen to satisfy

$$
E_{I}^{4}(t) \leq E_{I}^{4}(0)+C \epsilon_{0}^{5} N^{-3-\frac{3}{4}+} \text { for all } t \in[0,1] .
$$

Consequently, using (6.17), we see that the rescaled solution has

$$
\|I u(1)\|_{L^{2}}^{2}=\epsilon_{0}^{2}+O\left(\epsilon_{0}^{3}\right)+C \epsilon_{0}^{5} N^{-3-\frac{3}{4}+}<4 \epsilon_{0}^{2} .
$$

6.4. Iteration. We may now consider the initial value problem for $\mathrm{KdV}$ with initial data $u(1)$ and, in light of the preceding bound, the local result will advance the solution to time $t=2$. We iterate this process $M$ times and, in place of (6.18), we have

$$
E_{I}^{4}(t) \leq E_{I}^{4}(0)+M C \epsilon_{0}^{5} N^{-3-\frac{3}{4}+} \text { for all } t \in[0, M+1] .
$$

As long as $M N^{-3-\frac{3}{4}+} \lesssim 1$, we will have the bound

$$
I u(M)_{L^{2}}^{2}=\epsilon_{0}^{2}+O\left(\epsilon^{3}\right)+M C \epsilon_{0}^{5} N^{-3-\frac{3}{4}+}<4 \epsilon_{0}^{2},
$$

and the lifetime of the local results remains uniformly of size 1 . We take $M \sim$ $N^{3+\frac{3}{4}-}$. This process extends the local solution to the time interval $\left[0, N^{3+\frac{3}{4}-}\right]$. We choose $N=N(T)$ so that

$$
N^{3+\frac{3}{4}-}>\lambda^{3} T \sim N^{-\frac{6 s}{3+2 s}} T,
$$

which may certainly be done for $s>-\frac{3}{4}$. This completes the proof of global wellposedness for $K d V$ in $H^{s}(\mathbb{R}), s>-\frac{3}{4}$.

We make two observations regarding the rescalings of our global-in-time $\mathrm{KdV}$ solution:

$$
\begin{gathered}
\sup _{t \in[0, T]}\|u(t)\|_{H^{s}} \sim \lambda^{\frac{3}{2}+s} \sup _{t \in\left[0, \lambda^{3} T\right]}\left\|u_{\lambda}(t)\right\|_{H^{s}} \leq \lambda^{\frac{3}{2}+s} \sup _{t \in\left[0, \lambda^{3} T\right]}\left\|I u_{\lambda}(t)\right\|_{L^{2}}, \\
\left\|I \phi_{\lambda}\right\|_{L^{2}} \lesssim N^{-s}\left\|\phi_{\lambda}\right\|_{H^{s}} \sim N^{-s} \lambda^{-\frac{3}{2}-s}\|\phi\|_{H^{s}} .
\end{gathered}
$$

The almost conservation law and local well-posedness iteration argument presented above implies that provided $N$ and $\lambda$ are selected correctly

$$
\sup _{t \in\left[0, \lambda^{3} T\right]}\left\|I u_{\lambda}(t)\right\|_{L^{2}} \lesssim \sup _{t \in\left[0, \lambda^{3} T\right]}\left\|I \phi_{\lambda}\right\|_{L^{2}} .
$$

The estimate (6.21) forms a bridge between (6.19) and (6.20) which implies

$$
\sup _{t \in[0, T]}\|u(t)\|_{H^{s}} \lesssim N^{-s}\|\phi\|_{H^{s}} .
$$


In fact, the selection of $N$ is polynomial in the parameter $T$ so (6.22) gives a polynomial-in-time upper bound on $\|u(t)\|_{H^{s}}$.

The choice of $\lambda$. The parameter $\lambda$ was chosen above so that

$$
\left\|I \phi_{\lambda}\right\|_{L^{2}} \sim \epsilon_{0} \ll 1
$$

Since, from (6.20), $\left\|I \phi_{\lambda}\right\|_{L^{2}} \lesssim N^{-s} \lambda^{-\frac{3}{2}-s}\|\phi\|_{H^{s}}$, we see that (6.23) holds provided we choose

$$
\lambda=\lambda\left(N, \epsilon_{0},\|\phi\|_{H^{s}}\right) \sim\left(\frac{\|\phi\|_{H^{s}}}{\epsilon_{0}}\right)^{\frac{2}{3+2 s}} N^{-\frac{2 s}{3+2 s}} .
$$

The choice of $N$. The parameter $N$ is chosen so that

$$
N^{\beta}>\lambda^{3} T \sim c_{\|\phi\|_{H^{s}}, \epsilon_{0}} N^{-\frac{6 s}{3+2 s}} T,
$$

where $\beta$ is the exponent appearing in (5.6) (in the $\mathbb{R}$-case just presented, $\beta=$ $\left.3+\frac{3}{4}-\right)$. This unravels to give a sufficient choice of $N$ :

$$
N \sim c_{\|\phi\|_{H}, \epsilon_{0}} T^{\frac{3+2 s}{\beta(3+2 s)+6 s}} \sim c_{\|\phi\|_{H}, \epsilon_{0}} T^{\gamma(s)} .
$$

In the range $-\frac{3}{2}<s$, the numerator of the exponent on $T$ is positive. The denominator is positive provided $\beta>-\frac{6}{3+2 s}$. For $s=-\frac{3}{4},-\frac{6}{3+2 s}=3$ so we require better than third order decay with $N$ in the local-in-time increment (5.6). With $s=-\frac{3}{4}+, \beta=3+\frac{3}{4}-$, calculating $\gamma(s)$ and inserting the resulting expression for $N$ in terms of $T$ into (6.22) reveals that, for our global-in-time solutions of (1.1), we have

$$
\|u(t)\|_{H^{-\frac{3}{4}+}(\mathbb{R})} \lesssim t^{1+}\|\phi\|_{H^{-\frac{3}{4}+}} \cdot
$$

Remark 6.4. Observe that the polynomial exponent $1+$ in (6.27) does not explode as we approach the critical regularity value $-\frac{3}{4}$. This is due to the fact that (5.6) gave us much more decay than required for iterating the local result. In principle, the decay rate in (5.6) could be improved by going further along the sequence $\left\{E_{I}^{n}\right\}$ of modified energies.

\section{LOCAL WELL-POSEDNESS OF KDV ON $\mathbb{T}$}

This section revisits the local-in-time theory for periodic KdV developed by Kenig, Ponce and Vega [32] and Bourgain [5]. Our presentation provides details left unexposed in [32 and [5] and quantifies the dependence of various implied constants on the length of the spatial period. This quantification is necessary for the adaptation of the rescaling argument used in Section 6 to the periodic setting.

7.1. The $\lambda$-periodic initial value problem for $\mathbf{K d V}$. We consider the $\lambda$ periodic initial value problem for $K d V$ :

$$
\left\{\begin{array}{c}
\partial_{t} u+\partial_{x}^{3} u+\frac{1}{2} \partial_{x} u^{2}=0, \quad x \in[0, \lambda] \\
u(x, 0)=\phi(x)
\end{array}\right.
$$

We first want to build a representation formula for the solution of the linearization of (7.1) about the zero solution. So, we wish to solve the linear homogeneous $\lambda$-periodic initial value problem

$$
\left\{\begin{array}{l}
\partial_{t} w+\partial_{x}^{3} w=0, \quad x \in[0, \lambda] \\
w(x, 0)=\phi(x)
\end{array}\right.
$$


Define $(d k)_{\lambda}$ to be normalized counting measure on $\mathbb{Z} / \lambda$ :

$$
\int a(x)(d k)_{\lambda}=\frac{1}{\lambda} \sum_{k \in \mathbb{Z} / \lambda} a(k)
$$

Define the Fourier transform of a function $f$ defined on $[0, \lambda]$ by

$$
\widehat{f}(k)=\int_{0}^{\lambda} e^{-2 \pi i k x} f(x) d x
$$

and we have the Fourier inversion formula

$$
f(x)=\int e^{2 \pi i k x} \widehat{f}(k)(d k)_{\lambda} .
$$

The usual properties of the Fourier transform hold:

$$
\begin{gathered}
\|f\|_{L^{2}([0, \lambda])}=\|\widehat{f}\|_{L^{2}\left((d k)_{\lambda}\right)} \quad \text { (Plancherel), } \\
\int_{0}^{\lambda} f(x) \overline{g(x)} d x=\int \widehat{f}(k) \bar{g}(k)(d k)_{\lambda} \quad \text { (Parseval), } \\
\widehat{f g}(k)=\widehat{f} *_{\lambda} \widehat{g}(k)=\int \widehat{f}\left(k-k_{1}\right) \widehat{g}\left(k_{1}\right)\left(d k_{1}\right)_{\lambda} \quad \text { (Convolution), }
\end{gathered}
$$

and so on. If we apply $\partial_{x}^{m}, m \in \mathbb{N}$, to (7.5), we obtain

$$
\partial_{x}^{m} f(x)=\int e^{2 \pi i k x}(2 \pi i k)^{m} \widehat{f}(k)(d k)_{\lambda} .
$$

This, together with (7.6), motivates us to define the Sobolev space $H^{s}(0, \lambda)$ with the norm

$$
\|f\|_{H^{s}(0, \lambda)}=\left\|\widehat{f}(k)\langle k\rangle^{s}\right\|_{L^{2}\left((d k)_{\lambda}\right)} .
$$

We will often denote this space by $H^{s}$ for simplicity. Note that there are about $\lambda$ low frequencies in the range $|k| \lesssim 1$ where the $H^{s}$ norm consists of the $L^{2}$ norm.

The Fourier inversion formula (7.5) allows us to write down the solution of (7.2):

$$
w(x, t)=S_{\lambda}(t) \phi(x)=\int e^{2 \pi i k x} e^{-(2 \pi i k)^{3} t} \widehat{\phi}(k)(d k)_{\lambda} .
$$

For a function $v=v(x, t)$ which is $\lambda$-periodic with respect to the $x$ variable and with the time variable $t \in \mathbb{R}$, we define the space-time Fourier transform $\widehat{v}=\widehat{v}(k, \tau)$ for $k \in \mathbb{Z} / \lambda$ and $\tau \in \mathbb{R}$ by

$$
\widehat{v}(k, \tau)=\iint_{0}^{\lambda} e^{-2 \pi i k x} e^{-2 \pi i \tau t} v(x, t) d x d t .
$$

This transform is inverted by

$$
v(x, t)=\iint e^{2 \pi i k x} e^{2 \pi i \tau t} \widehat{v}(k, \tau)(d k)_{\lambda} d \tau .
$$

The expression (7.10) may be rewritten as a space-time inverse Fourier transform,

$$
S_{\lambda}(t) \phi(x)=\iint e^{2 \pi i k x} e^{2 \pi i \tau t} \delta\left(\tau-4 \pi^{2} k^{3}\right) \widehat{\phi}(k)(d k)_{\lambda} d \tau
$$

where $\delta(\eta)$ represents a 1 -dimensional Dirac mass at $\eta=0$. This recasting shows that $S_{\lambda}(\cdot) \phi$ has its space-time Fourier transform supported precisely on the cubic $\tau=4 \pi^{2} k^{3}$ in $\mathbb{Z} / \lambda \times \mathbb{R}$. 
We next find a representation for the solution of the linear inhomogeneous $\lambda$ periodic initial value problem

$$
\left\{\begin{array}{c}
\partial_{t} v+\partial_{x}^{3} v=f, \quad x \in[0, \lambda] \\
v(x, 0)=0
\end{array}\right.
$$

with $f=f(x, t)$ a given time-dependent $\lambda$-periodic (in $x)$ function. By Duhamel's principle,

$$
v(x, t)=\int_{0}^{t} S_{\lambda}\left(t-t^{\prime}\right) f\left(x, t^{\prime}\right) d t^{\prime} .
$$

We represent $f\left(x, t^{\prime}\right)$ using (7.12), apply (7.10) and rearrange integrations to find

$$
v(x, t)=\iint e^{2 \pi i k x} e^{2 \pi i\left(4 \pi^{2} k^{3} t\right)} \int_{0}^{t} e^{2 \pi i\left(\tau-4 \pi^{2} k^{3}\right) t^{\prime}} d t^{\prime} \widehat{f}(k, \tau)(d k)_{\lambda} d \tau .
$$

Performing the $t^{\prime}$-integration, we find

$$
v(x, t)=\iint e^{2 \pi k x} e^{2 \pi i\left(4 \pi^{2} k^{3} t\right)} \frac{e^{2 \pi i\left(\tau-4 \pi^{2} k^{3}\right) t}-1}{2 \pi i\left(\tau-4 \pi^{2} k^{3}\right)} \widehat{f}(k, \tau)(d k)_{\lambda} d \tau .
$$

The $\lambda$-periodic initial value problem for $\mathrm{KdV}(17.1)$ is equivalent to the integral equation

$$
u(t)=S_{\lambda}(t) \phi-\int_{0}^{t} S_{\lambda}\left(t-t^{\prime}\right)\left(\frac{1}{2} \partial_{x} u^{2}\left(t^{\prime}\right)\right) d t^{\prime} .
$$

Remark 7.1. The spatial mean $\int_{\mathbb{T}} u(x, t) d x$ is conserved during the evolution (7.1). We may assume that the initial data $\phi$ satisfies a mean-zero assumption $\int_{\mathbb{T}} \phi(x) d x$ since otherwise we can replace the dependent variable $u$ by $v=u-\int_{\mathbb{T}} \phi$ at the expense of a harmless linear first order term. This observation was used by Bourgain in [5]. The mean-zero assumption is crucial for some of the analysis that follows.

7.2. Spaces of functions of space-time. The integral equation (7.17) will be solved using the contraction principle in spaces introduced in this subsection. We also introduce some other spaces of functions of space-time which will be useful in our analysis of (17.17).

We define the $X_{s, b}$ spaces for $\lambda$-periodic $\mathrm{KdV}$ via the norm

$$
\|u\|_{X_{s, b}([0, \lambda] \times \mathbb{R})}=\left\|\langle k\rangle^{s}\left\langle\tau-4 \pi^{2} k^{3}\right\rangle^{b} \widehat{u}(k, \tau)\right\|_{L^{2}\left((d k)_{\lambda} d \tau\right)} .
$$

(We will suppress reference to the spatial period $\lambda$ in the notation for the space-time function spaces $X_{s, b}$ and the related spaces below.)

The study of periodic KdV in [32], [5] has been based around iteration in the spaces $X_{s, \frac{1}{2}}$. This space barely fails to control the $L_{t}^{\infty} H_{x}^{s}$ norm. To ensure continuity of the time flow of the solution we construct, we introduce the slightly smaller space $Y^{s}$ defined via the norm

$$
\|u\|_{Y^{s}}=\|u\|_{X_{s, \frac{1}{2}}}+\left\|\langle k\rangle^{s} \widehat{u}(k, \tau)\right\|_{L^{2}\left((d k)_{\lambda}\right) L^{1}(d \tau)} .
$$

If $u \in Y^{s}$, then $u \in L_{t}^{\infty} H_{x}^{s}$. We will construct the solution of (17.17) by proving a contraction estimate in the space $Y^{s}$. The mapping properties of (7.15) motivate the introduction of the companion spaces $Z^{s}$ defined via the norm

$$
\|u\|_{Z^{s}}=\|u\|_{X_{s,-\frac{1}{2}}}+\left\|\frac{\langle k\rangle^{s} \widehat{u}(k, \tau)}{\left\langle\tau-4 \pi^{2} k^{3}\right\rangle}\right\|_{L^{2}\left((d k)_{\lambda}\right) L^{1}(d \tau)} .
$$


Let $\eta \in C_{0}^{\infty}(\mathbb{R})$ be a nice bump function supported on $[-2,2]$ with $\eta=1$ on $[-1,1]$. It is easy to see that multiplication by $\eta(t)$ is a bounded operation on the spaces $Y^{s}, Z^{s}$, and $X_{s, b}$.

\subsection{Linear estimates.}

\section{Lemma 7.1.}

$$
\left\|\eta(t) S_{\lambda}(t) \phi\right\|_{Y^{s}} \lesssim\|\phi\|_{H^{s}} .
$$

The proof follows easily from the fact that

$$
\widehat{\eta S_{\lambda}(\phi)}(k, \tau)=\widehat{\phi}(k) \widehat{\eta}\left(\tau-4 \pi^{2} k^{3}\right) .
$$

Lemma 7.2.

$$
\left\|\eta(t) \int_{0}^{t} S_{\lambda}\left(t-t^{\prime}\right) F\left(t^{\prime}\right) d t^{\prime}\right\|_{Y^{s}} \lesssim\|F\|_{Z^{s}} .
$$

Proof. By applying a smooth cutoff, we may assume that $F$ is supported on $\mathbb{T} \times$ $[-3,3]$. Let $a(t)=\operatorname{sgn}(t) \tilde{\eta}(t)$, where $\tilde{\eta}$ is a smooth bump function supported on $[-10,10]$ which equals 1 on $[-5,5]$. The identity

$$
\chi_{[0, t]}\left(t^{\prime}\right)=\frac{1}{2}\left(a\left(t^{\prime}\right)-a\left(t-t^{\prime}\right)\right),
$$

valid for $t \in[-2,2]$ and $t^{\prime} \in[-3,3]$, allows us to rewrite $\eta(t) \int_{0}^{t} S\left(t-t^{\prime}\right) F\left(t^{\prime}\right) d t^{\prime}$ as a linear combination of

$$
\eta(t) S(t) \int_{\mathbb{R}} a\left(t^{\prime}\right) S\left(-t^{\prime}\right) F\left(t^{\prime}\right) d t^{\prime}
$$

and

$$
\eta(t) \int_{\mathbb{R}} a\left(t-t^{\prime}\right) S\left(t-t^{\prime}\right) F\left(t^{\prime}\right) d t^{\prime}
$$

Consider the contribution (7.24). By 7.21), it suffices to show that

$$
\left\|\int a\left(t^{\prime}\right) S\left(-t^{\prime}\right) F\left(t^{\prime}\right) d t^{\prime}\right\|_{H^{s}} \lesssim\|F\|_{Z^{s}} .
$$

Since the Fourier transform of $\int a\left(t^{\prime}\right) S\left(-t^{\prime}\right) F\left(t^{\prime}\right) d t^{\prime}$ evaluated at $\xi$ is given by $\int \widehat{a}\left(\tau-\xi^{3}\right) \widehat{F}(\xi, \tau) d \tau$ and one can easily verify that $|\widehat{a}(\tau)|=O\left(\langle\tau\rangle^{-1}\right)$, the claimed estimate follows using the definition (7.20).

For (7.25), we discard the cutoff $\eta(t)$ and note that the space-time Fourier transform of $\int a\left(t-t^{\prime}\right) S\left(t-t^{\prime}\right) F\left(t^{\prime}\right) d t^{\prime}$ evaluated at $(\xi, \tau)$ is equal to $\widehat{a}\left(\tau-4 \pi^{2} \xi^{3}\right) \widehat{F}(\xi, \tau)$. The claimed estimate then follows from the definitions (7.20), (7.19) and the decay estimate for $\widehat{a}$ used above.

Proposition 3. Let $\phi$ be a $\lambda$-periodic function whose Fourier transform is supported on $\{k:|k| \sim N\}$. Then

$$
\left\|\eta(t) S_{\lambda}(t) \phi\right\|_{L_{x, t}^{4}} \lesssim C(N, \lambda)\|\phi\|_{L_{x}^{2}}
$$

where

$$
C(N, \lambda)=\left\{\begin{array}{cl}
1 & \text { if } N \leq 1 \\
\left(\frac{1}{\sqrt{N}}+\frac{1}{\lambda}\right)^{\frac{1}{4}} & \text { if } N \geq 1 .
\end{array}\right.
$$


Remark 7.2. In the limit $\lambda \rightarrow \infty$, (7.26) yields the Strichartz estimate on the line (at least when $N \geq 1$ ),

$$
\left\|D_{x}^{\frac{1}{8}} e^{-t 4 \pi^{2} \partial_{x}^{3}} \phi\right\|_{L_{x \in \mathbb{R}, t}^{4}} \lesssim\|\phi\|_{L_{x \in \mathbb{R}}^{2}} .
$$

Proposition 3 (and the related Proposition 4 below) will not be used in our proof of local and global well-posedness of $\mathrm{KdV}$ on $\mathbb{T}$ but may be of relevance in studying other properties of the long period limit of the KdV equation.

Proof. It suffices to show that

$$
\left\|\left(\eta(t) S_{\lambda}(t) \phi_{1}\right)\left(\eta(t) S_{\lambda}(t) \phi_{2}\right)\right\|_{L_{x, t}^{2}}^{2} \leq C^{2}(N, \lambda)\left\|\phi_{1}\right\|_{L_{x}^{2}}\left\|\phi_{2}\right\|_{L_{x}^{2}}
$$

for functions $\phi_{1}, \phi_{2}$ satisfying the hypotheses. Properties of the Fourier transform allow us to re-express the left side as

$$
\left\|\int_{k=k_{1}+k_{2}, \tau=\tau_{1}+\tau_{2}} \widehat{\phi_{1}}\left(k_{1}\right) \widehat{\phi_{2}}\left(k_{2}\right) \psi\left(\tau_{1}-4 \pi^{2} k_{1}^{3}\right) \psi\left(\tau_{2}-4 \pi^{2} k_{2}^{3}\right)\left(d k_{1}\right)_{\lambda} d \tau_{1}\right\|_{L^{2}\left(d \tau(d k)_{\lambda}\right)}^{2},
$$

where $\psi=\widehat{\eta}$ may be take to be a positive even Schwarz function. We evaluate the $\tau_{1}$-integration by writing

$$
\int \psi\left(\tau_{1}-4 \pi^{2} k_{1}^{3}\right) \psi\left(\tau-\tau_{1}-4 \pi^{2} k_{2}^{3}\right) d \tau_{1}=\tilde{\psi}\left(\tau-4 \pi^{2} k_{1}^{3}-4 \pi^{2} k_{2}^{3}\right),
$$

with $\tilde{\psi}$ also rapidly decreasing. Inserting this into the re-expressed left side and applying Cauchy-Schwarz leads to the upper bound

$$
\begin{aligned}
& \|\left(\int \tilde{\psi}^{2}\left(\tau-4 \pi^{2} k_{1}^{3}-4 \pi^{2} k_{2}^{3}\right)\left(d k_{1}\right)_{\lambda}\right)^{\frac{1}{2}} \\
& \quad \times\left(\int \tilde{\psi}^{2}\left(\tau-4 \pi^{2} k_{1}^{3}-4 \pi^{2} k_{2}^{3}\right)\left|\widehat{\phi_{1}}\left(k_{1}\right)\right|^{2}\left|\widehat{\phi_{2}}\left(k_{2}\right)\right|^{2}\left(d k_{1}\right)_{\lambda}\right)^{\frac{1}{2}} \|_{L^{2}\left(d \tau(d k)_{\lambda}\right)} .
\end{aligned}
$$

The first integral may be pulled out of the $L^{2}$ norm and the $\tilde{\psi}^{2}$ term in the second integral is used to integrate in $\tau$ to give

$$
\lesssim\left\|\int \tilde{\psi}^{2}\left(\tau-4 \pi^{2} k_{1}^{3}-4 \pi^{2} k_{2}^{3}\right)\left(d k_{1}\right)_{\lambda}\right\|_{L_{k, \tau}^{\infty}}\left\|\phi_{1}\right\|_{L_{x}^{2}}\left\|\phi_{2}\right\|_{L_{x}^{2}}
$$

Matters are thus reduced to quantifying the $L^{\infty}$ norm above. Let $M$ denote $\left\|\int \tilde{\psi}^{2}\left(\tau-4 \pi^{2} k_{1}^{3}-4 \pi^{2} k_{2}^{3}\right)\left(d k_{1}\right)_{\lambda}\right\|_{L_{k, \tau}^{\infty}}$. We estimate $M$ by counting

$$
M \lesssim \frac{1}{\lambda}\left|\left\{k_{1} \in \mathbb{Z} / \lambda:\left|k_{1}\right| \sim N ;\left|k-k_{1}\right| \sim N ; k^{3}-3 k k_{1}\left(k-k_{1}\right)=\tau+O(1)\right\}\right| .
$$

In case $N \leq 1$, the cardinality of the set is $O(\lambda)$ so $C(N, \lambda) \lesssim 1$ for $N \leq 1$. Assume now that $N>1$ and rename $k_{1}=x$. The task is to estimate

$$
\left|\left\{x \in \mathbb{Z} / \lambda:|x|,|k-x| \sim N ; 3 k\left(x-\frac{k}{2}\right)^{2}-\frac{k^{2}}{4}=\tau-k^{3}+O(1)\right\}\right| .
$$

This set is largest when the parabola is the flattest, i.e., when $x \sim \frac{k}{2}$. We find that

$$
M \lesssim \frac{1}{\lambda}\left(\frac{1}{\sqrt{k}} \lambda+1\right)=\left(\frac{1}{\sqrt{k}}+\frac{1}{\lambda}\right)
$$

which completes the proof. 
Proposition 4. If $v=v(x, t)$ is a $\lambda$-periodic function of $x$ and the spatial Fourier transform of $v$ is supported on $\{k:|k| \sim N\}$, then

$$
\|\eta(t) v\|_{L_{x, t}^{4}} \lesssim C(N, \lambda)\|v\|_{X_{0, \frac{1}{2}+}},
$$

where $C(N, \lambda)$ is as it appears in (7.27).

This follows easily by stacking up cubic level sets on which (7.26) holds.

Lemma 7.3. If $v=v(x, t)$ is a $\lambda$-periodic function of $x$, then

$$
\|\eta(t) v\|_{L_{x, t}^{4}} \lesssim\|v\|_{X_{0, \frac{1}{3}}} .
$$

The estimate (7.31) is a rescaling of the $\lambda=1$ case proven by Bourgain [5].

Remark 7.3. We can interpolate between (7.30) and (7.31) to obtain

$$
\|\eta(t) v\|_{L_{x, t}^{4}} \lesssim\{C(N, \lambda)\}^{1-}\|v\|_{X_{0, \frac{1}{2}}} .
$$

\subsection{Bilinear estimate.}

Proposition 5. If $u$ and $v$ are $\lambda$-periodic functions of $x$, also depending upon $t$ having zero $x$-mean for all $t$, then

$$
\left\|\eta(t) \partial_{x}(u v)\right\|_{Z^{-\frac{1}{2}}} \lesssim \lambda^{0+}\|u\|_{X_{-\frac{1}{2}, \frac{1}{2}}}\|v\|_{X_{-\frac{1}{2}, \frac{1}{2}}}
$$

Note that (7.33) implies $\left\|\eta(t) \partial_{x}(u v)\right\|_{Z^{-\frac{1}{2}}} \lesssim \lambda^{0+}\|u\|_{Y^{-\frac{1}{2}}}\|v\|_{Y^{-\frac{1}{2}}}$. We will relax the notation by dispensing with various constants involving $\pi$ with the recognition that some of the formulas which follow may require adjusting the constants to be strictly correct.

Proof. The norms involved allow us to assume that $\widehat{u}$ and $\widehat{v}$ are nonnegative. There are two contributions to the $Z^{-\frac{1}{2}}$ norm we must control. We begin with the $X_{-\frac{1}{2},-\frac{1}{2}}$ contribution. Duality and an integration by parts shows that it suffices to prove

$$
\iint u_{1}(x, t) u_{2}(x, t) w_{x}(x, t) \eta(t) d x d t \lesssim \lambda^{0+}\left\|u_{1}\right\|_{X_{-\frac{1}{2}, \frac{1}{2}}}\left\|u_{2}\right\|_{X_{-\frac{1}{2}, \frac{1}{2}}}\|w\|_{X_{\frac{1}{2}, \frac{1}{2}}} .
$$

Writing $u_{3}=w_{x}$ shows that it suffices to prove

$$
\iint u_{1}(x, t) u_{2}(x, t) u_{3}(x, t) \eta(t) d x d t \lesssim \lambda^{0+}\left\|u_{1}\right\|_{X_{-\frac{1}{2}, \frac{1}{2}}}\left\|u_{2}\right\|_{X_{-\frac{1}{2}, \frac{1}{2}}}\left\|u_{3}\right\|_{X_{-\frac{1}{2}, \frac{1}{2}}}
$$

for all $u_{1}, u_{2}, u_{3}$ having zero $x$-mean. The left side may be rewritten

$$
\int_{k_{1}+k_{2}+k_{3}=0} \int_{\tau_{1}+\tau_{2}+\tau_{3}=0} \widehat{u_{1}}\left(k_{1}, \tau_{1}\right) \widehat{u_{2}}\left(k_{2}, \tau_{2}\right) \widehat{u_{3}}\left(k_{3}, \tau_{3}\right)\left(d k_{1}\right)_{\lambda}\left(d k_{2}\right)_{\lambda}\left(d k_{3}\right)_{\lambda} d \tau_{1} d \tau_{2} d \tau_{3} .
$$

Note that the mean zero conditions allow us to assume $k_{i} \neq 0$.

Case 1. $\left|k_{1}\right|,\left|k_{2}\right|,\left|k_{3}\right| \gtrsim 1$.

The identity

$$
3 k_{1} k_{2} k_{3}=k_{1}^{3}+k_{2}^{3}+k_{3}^{3}
$$

and the Case 1 defining conditions imply

$$
1 \lesssim \sum_{j=1}^{3} \frac{\left|\tau_{j}-k_{j}^{3}\right|}{\left\langle k_{1}\right\rangle\left\langle k_{2}\right\rangle\left\langle k_{3}\right\rangle}
$$


This of course implies

$$
1 \lesssim \sum_{j=1}^{3} \frac{\left\langle\tau_{j}-k_{j}^{3}\right\rangle^{\frac{1}{2}}}{\left\langle k_{1}\right\rangle^{\frac{1}{2}}\left\langle k_{2}\right\rangle^{\frac{1}{2}}\left\langle k_{3}\right\rangle^{\frac{1}{2}}}
$$

Inserting (7.36) into (7.35) and using symmetry reduces matters to showing that

$$
\begin{aligned}
& \int_{k_{1}+k_{2}+k_{3}=0} \int \widehat{\eta}\left(\tau_{1}+\tau_{2}+\tau_{3}\right) \frac{\widehat{u_{1}}\left(k_{1}, \tau_{1}\right)}{\left\langle k_{1}\right\rangle^{\frac{1}{2}}} \frac{\widehat{u_{2}}\left(k_{2}, \tau_{2}\right)}{\left\langle k_{2}\right\rangle^{\frac{1}{2}}} \frac{\widehat{u_{3}}\left(k_{3}, \tau_{3}\right)\left\langle\tau_{3}-k_{3}^{3}\right\rangle^{\frac{1}{2}}}{\left\langle k_{3}\right\rangle^{\frac{1}{2}}} \\
& \quad \lesssim \lambda^{0+}\left\|u_{1}\right\|_{X_{-\frac{1}{2}, \frac{1}{2}}}\left\|u_{2}\right\|_{X_{-\frac{1}{2}, \frac{1}{2}}}\left\|u_{3}\right\|_{X_{-\frac{1}{2}, \frac{1}{2}}}
\end{aligned}
$$

After some natural substitutions and undoing the Fourier transform, we see that it suffices to show that

$$
\left|\iint \eta(t) v_{1} v_{2} v_{3} d x d t\right| \lesssim \lambda^{0+}\left\|v_{1}\right\|_{X_{0, \frac{1}{2}}}\left\|v_{2}\right\|_{X_{0, \frac{1}{2}}}\left\|v_{3}\right\|_{X_{0,0}} .
$$

Using Hölder, we split the left side into $L_{x, t}^{4} L_{x, t}^{4} L_{x, t}^{2}$ and apply (7.31) to finish this case. (In fact, we control the left side of (7.37) with $\lambda^{0+}\left\|v_{1}\right\|_{X_{0, \frac{1}{3}}}\left\|v_{2}\right\|_{X_{0, \frac{1}{3}}}\left\|v_{3}\right\|_{X_{0,0}}$.)

Case 2. $\left|k_{1}\right|,\left|k_{2}\right|,\left|k_{3}\right| \lesssim 1$.

Derivatives are cheap in this frequency setting. We use Hölder to estimate (7.35) in $L^{4} L^{4} L^{2}$, and then we apply (7.31) to control the $L^{4}$ norms. Finally, we use the Case 2 defining conditions and Sobolev to move $X_{0, \frac{1}{3}}$ to $X_{-\frac{1}{2}, \frac{1}{2}}$ on two factors and $X_{0,0}$ to $X_{-\frac{1}{2}, \frac{1}{2}}$ on the remaining factor. (Again, we have encountered $X_{0, \frac{1}{3}}$ on two factors.)

Since $k_{1}+k_{2}+k_{3}=0$, the only remaining case to consider is when one of the frequencies is small and the other two are big. Symmetry permits us to focus on

Case 3. $0<\left|k_{3}\right| \lesssim 1 \lesssim\left|k_{1}\right|,\left|k_{2}\right|$.

The analog of (17.36) in this case is

$$
1 \lesssim\left|k_{3}\right|^{-\frac{1}{2}} \sum_{j=1}^{3} \frac{\left\langle\tau_{j}-k_{j}^{3}\right\rangle^{\frac{1}{2}}}{\left\langle k_{1}\right\rangle^{\frac{1}{2}}\left\langle k_{2}\right\rangle^{\frac{1}{2}}\left\langle k_{3}\right\rangle^{\frac{1}{2}}} .
$$

Since we are in the $\lambda$-periodic setting and our functions have zero $x$-mean, we have $\left|k_{3}\right| \gtrsim \frac{1}{\lambda}$. We analyze this expression in two cases: when the $j=3$ term dominates the $j=1, j=2$ terms and when the $j=1$ or $j=2$ term dominates the $j=3$ term. In case $j=3$ dominates, it suffices to prove

$$
\left|\int \eta(t) v_{1} \eta(t) v_{2} \eta(t) v_{3} d x d t\right| \lesssim \lambda^{0+}\left\|v_{1}\right\|_{X_{0, \frac{1}{2}}}\left\|v_{2}\right\|_{X_{0, \frac{1}{2}}}\left\|\left|\partial_{x}\right|^{\frac{1}{2}} v_{3}\right\|_{X_{0,0}} .
$$

(Strictly speaking, each $\eta$ that appears in (7.39) should be replaced by $\eta^{\frac{1}{3}}$ but we abuse the notation with the understanding that all smooth cutoff functions are essentially the same within this analysis.) The left side of (7.39) is estimated via Hölder by

$$
\left\|\eta(t) v_{1}\right\|_{L_{t}^{4} L_{x}^{2}}\left\|\eta(t) v_{2}\right\|_{L_{t}^{4} L_{x}^{2}}\left\|\eta(t) v_{3}\right\|_{L_{t}^{2} L_{x}^{\infty}}
$$

Since $X_{0, \frac{1}{2}}$ embeds into $L_{t}^{4} L_{x}^{2}$, the first two factors are fine. For the third factor, we estimate the $L_{x}^{\infty}$ norm

$$
\left\|v_{3}\right\|_{L_{x}^{\infty}} \leq \int_{\lambda^{-1} \lesssim\left|k_{3}\right| \lesssim 1}\left|k_{3}\right|^{-\frac{1}{2}}\left|k_{3}\right|^{\frac{1}{2}} \widehat{v_{3}}\left(k_{3}\right) d k_{3} \leq \lambda^{0+}\left\|\left|\partial_{x}\right|^{\frac{1}{2}} v_{3}\right\|_{L_{x}^{2}}
$$

and then take the $L_{t}^{2}$ (or $L_{\tau}^{2}$ ) norm. 
Consider next the case when the $j=1$ term dominates the $j=3$ term. (The case when $j=2$ dominates is similar.) It suffices to prove

$$
\left|\int \eta(t) v_{1} \eta(t) v_{2} \eta(t) v_{3} d x d t\right| \lesssim \lambda^{0+}\left\|v_{1}\right\|_{X_{0,0}}\left\|v_{2}\right\|_{X_{0, \frac{1}{2}}}\left\|\left|\partial_{x}\right|^{\frac{1}{2}} v_{3}\right\|_{X_{0, \frac{1}{2}}} .
$$

By Hölder, the left side is controlled by

$$
\left\|\eta(t) v_{1}\right\|_{L_{t}^{2} L_{x}^{2}}\left\|\eta(t) v_{2}\right\|_{L_{t}^{4} L_{x}^{2}}\left\|\eta(t) v_{3}\right\|_{L_{t}^{4} L_{x}^{\infty}} .
$$

The first two terms are easily bounded. For the third term, observe that

$$
\left\|\eta(t) v_{3}\right\|_{L_{t}^{2} L_{x}^{\infty}} \lesssim \lambda^{0+}\left\|\left|\partial_{x}\right|^{\frac{1}{2}} v_{3}\right\|_{X_{0,0}}
$$

and

$$
\left\|\eta(t) v_{3}\right\|_{L_{t}^{\infty} L_{x}^{\infty}} \lesssim \lambda^{0+}\left\|\left|\partial_{x}\right|^{\frac{1}{2}} v_{3}\right\|_{X_{0, \frac{1}{2}+}} .
$$

By interpolation, the desired inequality (17.40) follows.

The preceding discussion established that

$$
\left\|\frac{\langle k\rangle^{\frac{1}{2}}}{\left\langle\tau-k^{3}\right\rangle^{\frac{1}{2}}} \widehat{u_{1} u_{2}}(k, \tau)\right\|_{L_{k}^{2} L_{\tau}^{2}} \lesssim \lambda^{0+}\left\|u_{1}\right\|_{X_{-\frac{1}{2}, \frac{1}{2}}}\left\|u_{2}\right\|_{X_{-\frac{1}{2}, \frac{1}{2}}} .
$$

It remains to control the weighted $L_{k}^{2} L_{\tau}^{1}$ portion of the $Z^{-\frac{1}{2}}$ norm to complete the proof of (7.33). Since $\left|\langle k\rangle^{-\frac{1}{2}} \widehat{\partial_{x}(u v)}(k, \tau)\right| \sim\langle k\rangle^{\frac{1}{2}}|\widehat{u v}(k, \tau)|$, it suffices to prove that

$$
\left\|\frac{\langle k\rangle^{\frac{1}{2}}}{\left\langle\tau-k^{3}\right\rangle} \widehat{u_{1} u_{2}}(k, \tau)\right\|_{L_{k}^{2} L_{\tau}^{1}} \lesssim \lambda^{0+}\left\|u_{1}\right\|_{X_{-\frac{1}{2}, \frac{1}{2}}}\left\|u_{2}\right\|_{X_{-\frac{1}{2}, \frac{1}{2}}} .
$$

The left side of (7.42) may be rewritten

$$
\left\|\langle k\rangle^{\frac{1}{2}}\right\| \frac{1}{\left\langle\tau-k^{3}\right\rangle} \widehat{u_{1} u_{2}}(k, \tau)\left\|_{L_{\tau}^{1}}\right\|_{L_{k}^{2}} .
$$

The desired estimate may be re-expressed as

$$
\begin{aligned}
& \| \int_{k=k_{1}+k_{2}} \int_{\tau=\tau_{1}+\tau_{2}} \frac{\langle k\rangle^{\frac{1}{2}}\left\langle k_{1}\right\rangle^{\frac{1}{2}}\left\langle k_{2}\right\rangle^{\frac{1}{2}}}{\left\langle\tau-k^{3}\right\rangle\left\langle\tau_{1}-k_{1}^{3}\right\rangle^{\frac{1}{2}}\left\langle\tau_{2}-k_{2}^{3}\right\rangle^{\frac{1}{2}}} \widehat{u_{1}}\left(k_{1}, \tau_{1}\right) \widehat{u_{2}}\left(k_{2}, \tau_{2}\right) \\
& \quad \lesssim \lambda_{L_{k}^{2} L_{\tau}^{1}} \\
& \quad\left\|u_{1}\right\|_{X_{0,0}}\left\|u_{2}\right\|_{X_{0,0}} .
\end{aligned}
$$

Recall that the $L_{k}^{2}$ norm and the various $k$-integrations are with respect to the $\lambda$-dependent measure $(d k)_{\lambda}$.

Since we may assume our functions have mean zero, we have that $k k_{1} k_{2} \neq 0$ and the identity

$$
\tau-k^{3}=\left(\tau_{1}-k_{1}^{3}\right)+\left(\tau_{2}-k_{2}^{3}\right)-3 k k_{1} k_{2}
$$

implies that

$$
\max \left(\left\langle\tau-k^{3}\right\rangle,\left\langle\tau_{1}-k_{1}^{3}\right\rangle,\left\langle\tau_{1}-k_{1}^{3}\right\rangle\right) \gtrsim\left|k k_{1} k_{2}\right| .
$$

In case $\left\langle\tau_{1}-k_{1}^{3}\right\rangle$ is that maximum, we reduce matters to (7.41). Indeed, we rewrite 
(7.44) as

$$
\begin{aligned}
& \left\|\left\langle\tau-k^{3}\right\rangle^{-\frac{2}{3}}\left\langle\tau-k^{3}\right\rangle^{-\frac{1}{3}} \int_{k=k_{1}+k_{2}} \int_{\tau=\tau_{1}+\tau_{2}} \widehat{u_{1}}\left(k_{1}, \tau_{1}\right) \widehat{v_{2}}\left(k_{2}, \tau_{2}\right)\right\|_{L_{k}^{2} L_{\tau}^{1}} \\
& \quad \lesssim \lambda^{0+}\left\|u_{1}\right\|_{X_{0,0}}\left\|v_{2}\right\|_{X_{0, \frac{1}{2}}} \cdot
\end{aligned}
$$

Cauchy-Schwarz in $\tau$ (with the observation that $2\left(-\frac{2}{3}\right)<-1$ ) reduces this case to proving

$$
\left\|\left\langle\tau-k^{3}\right\rangle^{-\frac{1}{3}} \int_{k=k_{1}+k_{2}} \int_{\tau=\tau_{1}+\tau_{2}} \widehat{u_{1}}\left(k_{1}, \tau_{1}\right) \widehat{u_{2}}\left(k_{2}, \tau_{2}\right)\right\|_{L_{k}^{2} L_{\tau}^{2}} \lesssim \lambda^{0+}\left\|u_{1}\right\|_{X_{0,0}}\left\|u_{2}\right\|_{X_{0, \frac{1}{2}}} .
$$

Upon rewriting the left side using duality, we see that an $L_{x t}^{4} L_{x t}^{2} L_{x t}^{4}$ Hölder application using (7.31) finishes off this case. The situation when $\left\langle\tau_{2}-k_{2}^{3}\right\rangle$ is the maximum is symmetric so we are reduced to considering the case when $\left\langle\tau-k^{3}\right\rangle$ is the maximum in (7.46).

In the event that

$$
\left\langle\tau_{1}-k_{1}^{3}\right\rangle \gtrsim\left|k k_{1} k_{2}\right|^{\frac{1}{100}}
$$

we get a little help from the 1-denominator in (17.44). We cancel $\left\langle\tau_{1}-k_{1}^{3}\right\rangle^{\frac{1}{6}}$ leaving $\left\langle\tau_{1}-k_{1}^{3}\right\rangle^{\frac{1}{3}}$ in the denominator and $\left(\langle k\rangle\left\langle k_{1}\right\rangle\left\langle k_{2}\right\rangle\right)^{\frac{1}{2}-}$ in the numerator. After the natural cancellation using (7.46), we collapse to needing to prove

$$
\left\|\left\langle\tau-k^{3}\right\rangle^{-\frac{1}{2}-} \int_{k=k_{1}+k_{2}} \int_{\tau=\tau_{1}+\tau_{2}} \widehat{u_{1}}\left(k_{1}, \tau_{1}\right) \widehat{u_{2}}\left(k_{2}, \tau_{2}\right)\right\|_{L_{k}^{2} L_{\tau}^{1}} \lesssim \lambda^{0+}\left\|u_{1}\right\|_{X_{0, \frac{1}{3}}}\left\|u_{2}\right\|_{X_{0, \frac{1}{2}}} .
$$

We apply Cauchy-Schwarz in $\tau$ to obtain the upper bound

$$
\left\|\int_{k=k_{1}+k_{2}} \int_{\tau=\tau_{1}+\tau_{2}} \widehat{u_{1}}\left(k_{1}, \tau_{1}\right) \widehat{u_{2}}\left(k_{2}, \tau_{2}\right)\right\|_{L_{k}^{2} L_{\tau}^{2}}
$$

which is controlled as desired using (7.31). The case when $\left\langle\tau_{2}-k_{2}^{3}\right\rangle \gtrsim\left|k k_{1} k_{2}\right|^{\frac{1}{100}}$ is symmetric.

All that remains is the situation when

$$
\left\langle\tau_{i}-k_{i}^{3}\right\rangle \ll\left|k k_{1} k_{2}\right|^{\frac{1}{100}}, \quad i=1,2 .
$$

Recalling (7.45), we see here that

$$
\tau-k^{3}=-3 k k_{1} k_{2}+O\left(\left\langle k k_{1} k_{2}\right\rangle^{\frac{1}{100}}\right),
$$

which we use to restrict $\tau$. After performing the natural cancellation using (7.46) on (7.44), we wish to show that

$$
\begin{aligned}
& \left\|\left\langle\tau-k^{3}\right\rangle^{-\frac{1}{2}} \int_{k=k_{1}+k_{2}} \int_{\tau=\tau_{1}+\tau_{2}} \widehat{u_{1}}\left(k_{1}, \tau_{1}\right) \widehat{u_{2}}\left(k_{2}, \tau_{2}\right) \chi_{\Omega(k)}\left(\tau-k^{3}\right)\right\|_{L_{k}^{2} L_{\tau}^{1}} \\
& \quad \lesssim \lambda^{0+}\left\|u_{1}\right\|_{X_{0, \frac{1}{2}}}\left\|u_{2}\right\|_{X_{0, \frac{1}{2}}},
\end{aligned}
$$


where the set

$$
\begin{aligned}
\Omega(k)=\left\{\eta \in \mathbb{R}: \eta=-3 k k_{1} k_{2}+O\left(\left\langle k k_{1} k_{2}\right\rangle \frac{1}{100}\right)\right. \\
\\
\left.\quad \text { for any } k_{1}, k_{2} \in \mathbb{Z} / \lambda \text { with } k=k_{1}+k_{2}\right\} .
\end{aligned}
$$

We apply Cauchy-Schwarz in $\tau$ to bound the left side of (7.49) by

$$
\left\|\left(\int\left\langle\tau-k^{3}\right\rangle^{-1} \chi_{\Omega(k)}\left(\tau-k^{3}\right) d \tau\right)^{\frac{1}{2}}\right\|_{k=k_{1}+k_{2}} \int_{\tau=\tau_{1}+\tau_{2}} \widehat{u_{1}}\left(k_{1}, \tau_{1}\right) \widehat{u_{2}}\left(k_{2}, \tau_{2}\right)\left\|_{L_{\tau}^{2}}\right\|_{L_{k}^{2}} .
$$

The point here is that the characteristic function appearing in the $\tau$-integrand above sufficiently restricts the region of integration to prove

$$
\left(\int\left\langle\tau-k^{3}\right\rangle^{-1} \chi_{\Omega(k)}\left(\tau-k^{3}\right) d \tau\right)^{\frac{1}{2}} \lesssim C+\lambda^{0+}
$$

uniformly in the parameter $k$. Note that familiar arguments complete the proof of (7.49) (and, hence, (7.42) provided we show (7.50).

Remark 7.4. The condition (7.48) restricts the functions $\widehat{u_{i}}$ essentially to the dispersive curve $\left\{\left(k, k^{3}\right): k \in \mathbb{Z} / \lambda\right\}$. Suppose for the moment that $\lambda=1$ and we restrict our attention to those $k$ satisfying $|k| \sim N$. Observe that the projection of the point set $S_{N}=\left\{\left(k, k^{3}\right) \in \mathbb{Z}_{k} \times \mathbb{R}_{\tau}: k \in \mathbb{Z},|k| \sim N\right\}$ onto the $\tau$-axis is a set of $N$ points which are $N^{2}$-separated. Therefore, if we "vertically thicken" these points $O\left(N^{\alpha}\right)$ for $\alpha \ll 2$, the projected set remains rather sparse on the $\tau$-axis. The intuition underlying the proof of (77.50) is that a vertical thickening of the set $S_{N_{1}}+S_{N_{2}}$ also projects onto a thin set on the $\tau$ axis.

Lemma 7.4. Fix $k \in \mathbb{Z} \backslash\{0\}$. For $k_{1}, k_{2} \in \mathbb{Z} \backslash\{0\}$, we have for all dyadic $M \geq 1$ that

$$
\left|\left\{\mu \in \mathbb{R}:|\mu| \sim M, \mu=-3 k k_{1} k_{2}+O\left(\left\langle k k_{1} k_{2}\right\rangle^{\frac{1}{100}}\right)\right\}\right| \lesssim M^{1-\delta}
$$

for some $\delta>0$.

Proof. The hypotheses are symmetric in $k_{1}, k_{2}$ so we may assume $\left|k_{1}\right| \geq\left|k_{2}\right|$. We first consider the situation when $|k| \geq\left|k_{1}\right|$. The expression

$$
\mu=-3 k k_{1} k_{2}+O\left(\left\langle k k_{1} k_{2}\right\rangle^{\frac{1}{100}}\right)
$$

allows us to conclude that $|k| \lesssim|\mu| \lesssim|k|^{3}$ since $k_{1}, k_{2} \in \mathbb{Z} \backslash\{0\}$ and $\left|k k_{1} k_{2}\right| \lesssim|k|^{3}$. Suppose $|\mu| \sim M$ (dyadic) and $|k| \sim N$ (dyadic). We have, for some $p \in[1,3]$, that $M \sim N^{p}$. For $\mu$ to satisfy (17.52), $\left|k_{1} k_{2}\right| \sim M^{1-\frac{1}{p}}$. We make the crude observation that there are at most $M^{1-\frac{1}{p}}$ multiples of $M^{\frac{1}{p}}$ in the dyadic block $\{|\mu| \sim M\}$. Hence, the set of possible $\mu$ satisfying (7.52) must lie inside a union of $M^{1-\frac{1}{p}}$ intervals of size $M^{\frac{1}{100}}$, each of which contains an integer multiple of $k$. We have then that

$$
\left|\left\{\mu \in \mathbb{R}:|\mu| \sim M, \mu=-3 k k_{1} k_{2}+O\left(\left\langle k k_{1} k_{2}\right\rangle^{\frac{1}{100}}\right)\right\}\right|<M^{1-\frac{1}{p}} M^{\frac{1}{100}} \lesssim M^{\frac{3}{4}},
$$

since $1 \leq p \leq 3$.

In case $|k| \leq\left|k_{1}\right|$, we must have $\left|k_{1}\right| \lesssim|\mu| \lesssim\left|k_{1}\right|^{3}$ so, if $\left|k_{1}\right| \sim N_{1}$ (dyadic), we must have $M \sim N_{1}^{p}$ for some $p \in[1,3]$ and we can repeat the argument presented above. 
Remark 7.5. If we change the setting of the lemma to the case where $k, k_{1}, k_{2} \in$ $\mathbb{Z} / \lambda \backslash\{0\}$, we have to adjust the conclusion to read

$$
\left|\left\{\mu \in \mathbb{R}:|\mu| \sim M, \mu=-3 k k_{1} k_{2}+O\left(\left\langle k k_{1} k_{2}\right\rangle^{\frac{1}{100}}\right)\right\}\right| \lesssim \lambda^{1} M^{1-\delta}, \quad \delta>0 .
$$

We use the lemma to prove (7.50). A change of variables leads us to consider

$$
\int\langle\mu\rangle^{-1} \chi_{\Omega(k)}(\mu) d \mu \text {. }
$$

We decompose the integration and use (7.53):

$$
\begin{aligned}
& =\int_{|\mu|<\lambda^{1000}}\langle\mu\rangle^{-1} \chi_{\Omega(k)}(\mu) d \mu+\sum_{M: \lambda^{1000}<M \text { (dyadic) }} \int_{|\mu| \sim M}\langle\mu\rangle^{-1} \chi_{\Omega(k)}(\mu) d \mu \\
& \leq 1000 \log \lambda+\sum_{M: \lambda^{1000}<M \text { (dyadic) }} M^{-1} M^{1-\delta} \lambda^{1} .
\end{aligned}
$$

Finally, we crush $\lambda^{1}$ using the extra decay in $M$ to obtain

$$
\lesssim \log \lambda+\sum_{M(\text { dyadic })} M^{-\frac{\delta}{2}}
$$

which proves (7.50).

7.5. Contraction. Consider the $\lambda$-periodic initial value problem (7.1) with periodic initial data $\phi \in H^{s}(0, \lambda), s \geq-\frac{1}{2}$. We show first that, for arbitrary $\lambda$, this problem is well-posed on a time interval of size $\sim 1$ provided $\|\phi\|_{H^{-\frac{1}{2}(0, \lambda)}}$ is sufficiently small. Then we show by a rescaling argument that (7.1) is locally well-posed for arbitrary initial data $\phi \in H^{s}(0, \lambda)$.

As mentioned before in Remark [7.1, we restrict our attention to initial data having zero $x$-mean.

Fix $\phi \in H^{s}(0, \lambda), s \geq-\frac{1}{2}$ and for $w \in Z^{-\frac{1}{2}}$ define

$$
\Phi_{\phi}[w]=\eta(t) S_{\lambda}(t) \phi-\eta(t) \int_{0}^{t} S_{\lambda}\left(t-t^{\prime}\right)\left(\eta\left(t^{\prime}\right) w\left(t^{\prime}\right)\right) d t^{\prime} .
$$

The bilinear estimate (7.33) shows that $u \in Y^{-\frac{1}{2}}$ implies $\eta(t) \partial_{x}\left(u^{2}\right) \in Z^{-\frac{1}{2}}$ so the (nonlinear) operator

$$
\Gamma(u)=\Phi_{\phi}\left(\eta(t) \frac{1}{2} \partial_{x}\left(u^{2}\right)\right)
$$

is defined on $Y^{-\frac{1}{2}}$. Observe that $\Gamma(u)=u$ is equivalent, at least for $t \in[-1,1]$, to (7.17), which is equivalent to (7.1).

Claim 1. $\Gamma$ : (bounded subsets of $\left.Y^{-\frac{1}{2}}\right) \longmapsto\left(\right.$ bounded subsets of $Y^{-\frac{1}{2}}$ ).

We estimate

$$
\|\Gamma(u)\|_{Y^{-\frac{1}{2}}} \leq\left\|S_{\lambda}(t) \phi\right\|_{Y^{-\frac{1}{2}}}+\left\|\eta(t) \int_{0}^{t} S_{\lambda}\left(t-t^{\prime}\right)\left(\eta\left(t^{\prime}\right) \frac{1}{2} \partial_{x} u^{2}\left(t^{\prime}\right)\right) d t^{\prime}\right\|_{Y^{-\frac{1}{2}}} .
$$

By (7.21) and (7.23), followed by the bilinear estimate (7.33),

$$
\leq C_{1}\|\phi\|_{H^{s}(0, \lambda)}+C_{2}\left\|\eta(t) \partial_{x} u^{2}\right\|_{Z^{-\frac{1}{2}}} \leq C_{1}\|\phi\|_{H^{s}(0, \lambda)}+C_{2} C_{3} \lambda^{0+}\|u\|_{Y^{-\frac{1}{2}}}^{2}
$$

and the claim is proven. 
Consider the ball

$$
B=\left\{u \in Y^{-\frac{1}{2}}:\|w\|_{Y^{-\frac{1}{2}}} \leq C_{4}\|\phi\|_{H^{-\frac{1}{2}}(0, \lambda)}\right\} .
$$

Claim 2. $\Gamma$ is a contraction on $B$ if $\|\phi\|_{H^{-\frac{1}{2}(0, \lambda)}}$ is sufficiently small.

We wish to prove that for some $\theta \in(0,1)$,

$$
\|\Gamma(u)-\Gamma(v)\|_{Y^{-\frac{1}{2}}} \leq \theta\|u-v\|_{Y^{-\frac{1}{2}}}
$$

for all $u, v \in B$. Since $u^{2}-v^{2}=(u+v)(u-v)$, it is not difficult to see that

$$
\|\Gamma(u)-\Gamma(v)\|_{Y^{-\frac{1}{2}}} \leq C_{2} C_{3} \lambda^{0+}\left(\|u\|_{Y^{-\frac{1}{2}}}+\|v\|_{Y^{-\frac{1}{2}}}\right)\|u-v\|_{Y^{-\frac{1}{2}}} .
$$

Since $u, v \in B$,

$$
\|\Gamma(u)-\Gamma(v)\|_{Y^{-\frac{1}{2}}} \leq \lambda^{0+}\|\phi\|_{H^{-\frac{1}{2}}(0, \lambda)}\|u-v\|_{Y^{-\frac{1}{2}}} .
$$

Hence, for fixed $\lambda$, if we take $\phi$ so small that

$$
\lambda^{0+}\|\phi\|_{H^{-\frac{1}{2}}(0, \lambda)} \ll 1
$$

the contraction estimate is verified.

The preceding discussion establishes well-posedness of (7.1) on a $O(1)$-sized time interval for any initial data satisfying (17.54) .

Finally, consider (7.1) with $\lambda=\lambda_{0}$ fixed and $\phi \in H^{s}\left(0, \lambda_{0}\right), s \geq-\frac{1}{2}$. This problem is well-posed on a small time interval $[0, \delta]$ if and only if the $\sigma$-rescaled problem

$$
\left\{\begin{array}{c}
\partial_{t} u_{\sigma}+\partial_{x}^{3} u_{\sigma}+\frac{1}{2} \partial_{x} u_{\sigma}^{2}=0, \quad x \in\left[0, \sigma \lambda_{0}\right] \\
u_{\sigma}(x, 0)=\sigma^{-2} \phi\left(\frac{x}{\sigma}\right)
\end{array}\right.
$$

is well-posed on $\left[0, \sigma^{3} \delta\right]$. A simple calculation shows that

$$
\left\|\phi_{\sigma}\right\|_{H^{-\frac{1}{2}}\left(0, \sigma \lambda_{0}\right)}=\sigma^{-1}\|\phi\|_{H^{-\frac{1}{2}}\left(0, \lambda_{0}\right)} .
$$

Observe that

$$
\left(\sigma \lambda_{0}\right)^{0+}\left\|\phi_{\sigma}\right\|_{H^{-\frac{1}{2}}\left(0, \sigma \lambda_{0}\right)} \leq\left(\sigma \lambda_{0}\right)^{0+} \sigma^{-1}\|\phi\|_{H^{-\frac{1}{2}}\left(0, \lambda_{0}\right)} \ll 1
$$

provided $\sigma=\sigma\left(\lambda_{0},\|\phi\|_{H^{-\frac{1}{2}}\left(0, \lambda_{0}\right)}\right)$ is taken to be sufficiently large. This verifies (7.54) for the problem (7.55) proving well-posedness of (7.55) on the time interval, say $[0,1]$. Hence, (7.1) is locally well-posed for $t \in\left[0, \sigma^{-3}\right]$.

The preceding discussion reproves the local well-posedness result for periodic $\mathrm{KdV}$ in [32]. We record the following simple variant which will be used in proving the global result for (1.2). See Section 11 of [17] for a general interpolation lemma related to this proposition.

Proposition 6. If $s \geq-\frac{1}{2}$, the initial value problem (1.2) is locally well-posed for data $\phi$ satisfying $I \phi \in L^{2}(\mathbb{T})$. Moreover, the solution exists on a time interval $[0, \delta]$ with the lifetime

$$
\delta \sim\|I \phi\|_{L^{2}}^{-\alpha}
$$

and the solution satisfies the estimate

$$
\|I u\|_{Y^{0}} \lesssim\|I \phi\|_{L^{2}} .
$$




\section{Almost conservation and global well-posedness of KdV on $\mathbb{T}$}

This section proves that the 1-periodic initial value problem (7.1) for $\mathrm{KdV}$ is globally well-posed for initial data $\phi \in H^{s}(\mathbb{T})$ provided $s \geq-\frac{1}{2}$. In particular, we prove Theorem 2. The proof is an adaptation of the argument presented for the real line to the periodic setting.

8.1. Quintilinear estimate. The following quintilinear space-time estimate controls the increment of the modified energy $E_{I}^{4}$ during the lifetime of the local wellposedness result.

Lemma 8.1. Let $w_{i}=w_{i}(x, t)$ be $\lambda$-periodic function in $x$ also depending upon $t$. Let $\mathbb{P}$ denote the orthogonal projection onto mean zero functions, $\mathbb{P} u(x)=u(x)-$ $\int_{0}^{\lambda} u(y) d y$. Assume that $\int_{0}^{\lambda} w_{i}(x, t) d x=0$ for all $t$. Then

$$
\left|\int_{0}^{\delta} \int_{0}^{\lambda} \mathbb{P}\left(u_{1} u_{2} u_{3}\right) u_{4} u_{5} d x d t\right| \lesssim \lambda^{0+} \prod_{j=1}^{3}\left\|w_{j}\right\|_{Y^{\frac{1}{2}}}\left\|w_{4}\right\|_{X_{-\frac{1}{2}, \frac{1}{2}}}\left\|w_{5}\right\|_{X_{-\frac{1}{2}, \frac{1}{2}}} .
$$

Proof (apart from the endpoint). We group $w_{1}, w_{2}, w_{3}$ together and apply (7.34) to control the left side by

$$
\lambda^{0+}\left\|\mathbb{P}\left(w_{1} w_{2} w_{3}\right)\right\|_{X_{-\frac{1}{2}, \frac{1}{2}}}\left\|w_{4}\right\|_{X_{-\frac{1}{2}, \frac{1}{2}}}\left\|w_{5}\right\|_{X_{-\frac{1}{2}, \frac{1}{2}}} .
$$

The quintilinear estimate (8.1) is thus reduced to proving the trilinear estimate

$$
\left\|w_{1} w_{2} w_{3}\right\|_{X_{-\frac{1}{2}, \frac{1}{2}}} \lesssim \lambda^{0+}\left\|w_{1}\right\|_{Y^{\frac{1}{2}}}\left\|w_{2}\right\|_{Y^{\frac{1}{2}}}\left\|w_{3}\right\|_{Y^{\frac{1}{2}}} .
$$

The estimate 8.2 is implied by the more general fact: For any $s \geq \frac{1}{2}$,

$$
\left\|\prod_{i=1}^{k} u_{i}\right\|_{X_{s-1, \frac{1}{2}}} \lesssim \prod_{i=1}^{k}\left\|u_{i}\right\|_{Y^{s}} .
$$

The multilinear estimate (8.3) is proved in the forthcoming paper [17]. Here we indicate the proof for the $k=3$ case of (8.3), namely (8.2), when $s \in\left(\frac{1}{2}, 1\right]$. The proof for $s=\frac{1}{2}$ in [17] supplements the discussion presented below with some elementary number theory. The reader willing to accept (8.2) may proceed to Lemma 8.2 .

The Fourier transform of $\prod_{i=1}^{3} \widehat{u_{i}}(x, t)$ equals

$$
\int_{*} \prod_{i=1}^{3} \widehat{u_{i}}\left(k_{i}, \tau_{i}\right)
$$

where $\int_{*}$ denotes an integration over the set where $k=k_{1}+k_{2}+k_{3}, \tau=\tau_{1}+\tau_{2}+\tau_{3}$. We make a case-by-case analysis by decomposing the left side of (8.2) into various regions. We may assume that $\widehat{u}_{i}, i=1,2,3$, are nonnegative $\mathbb{R}$-valued functions.

Case 1. $\left\langle\tau-k^{3}\right\rangle \lesssim\left\langle\tau_{1}-k_{1}^{3}\right\rangle$.

In this case, it suffices to show that

$$
\left\|\prod_{i=1}^{3} u_{i}\right\|_{X_{s-1,0}} \lesssim\left\|u_{1}\right\|_{X_{s, 0}}\left\|u_{2}\right\|_{Y^{s}}\left\|u_{3}\right\|_{Y^{s}} .
$$


We observe using Sobolev that

$$
\left\|\prod_{i=1}^{3} u_{i}\right\|_{X_{s-1,0}} \lesssim\left\|\prod_{i=1}^{3} u_{i}\right\|_{L_{t}^{2} L_{x}^{1+}},
$$

and then, by Hölder,

$$
\lesssim\left\|u_{1}\right\|_{L_{t}^{2} L_{x}^{3+}}\left\|u_{1}\right\|_{L_{t}^{\infty} L_{x}^{3+}}\left\|u_{1}\right\|_{L_{t}^{\infty} L_{x}^{3+}}
$$

Finally, using Sobolev again and the embedding $Y^{s} \subset L_{t}^{\infty} H_{x}^{s}$, we conclude that (8.5) holds. Since $\left\langle\tau_{j}-k_{j}^{3}\right\rangle \gtrsim\left\langle\tau-k^{3}\right\rangle$ for $j=2,3$ is symmetric with the Case 1 defining condition, we may assume that we are in Case 2.

Case 2. $\left\langle\tau_{i}-k_{i}^{3}\right\rangle \ll\left\langle\tau-k^{3}\right\rangle$ for $i=1,2,3$.

The convolution constraints $k=k_{1}+k_{2}+k_{3}, \tau=\tau_{1}+\tau_{2}+\tau_{3}$ in this case imply that

$$
1 \ll\left\langle\tau-k^{3}\right\rangle \sim\left|k^{3}-\left(k_{1}^{3}+k_{2}^{3}+k_{3}^{3}\right)\right| .
$$

Therefore, it suffices to show that

$$
\begin{aligned}
\left\|\int_{*}\langle k\rangle^{s-1}\left|k^{3}-\left(k_{1}^{3}+k_{2}^{3}+k_{3}^{3}\right)\right|^{\frac{1}{2}} \widehat{u_{1}}\left(k_{1}, \tau_{1}\right) \widehat{u_{2}}\left(k_{2}, \tau_{2}\right) \widehat{u_{3}}\left(k_{3}, \tau_{3}\right)\right\|_{L_{\tau}^{2} L_{k}^{2}} \\
\quad \lesssim \prod_{i=1}^{3}\left\|u_{i}\right\|_{X_{s, \frac{1}{2}}} .
\end{aligned}
$$

This estimate may be recast by wiggling the weights and using duality as

$$
\begin{aligned}
\int_{*} \widehat{f}_{4}(k, \tau) \frac{\left|k^{3}-\left(k_{1}^{3}+k_{2}^{3}+k_{3}^{3}\right)\right|^{\frac{1}{2}}}{\langle k\rangle^{1-s} \prod_{i=1}^{3}\left\langle k_{i}\right\rangle^{s}\left\langle\tau_{i}-k_{i}^{3}\right\rangle^{\frac{1}{2}}} \widehat{f}_{1}\left(k_{1}, \tau_{1}\right) \widehat{f}_{2}\left(k_{2}, \tau_{2}\right) \widehat{f}_{3}\left(k_{3}, \tau_{3}\right) \\
\quad \lesssim \prod_{i=1}^{4}\left\|f_{i}\right\|_{L_{\tau}^{2} L_{k}^{2} .}
\end{aligned}
$$

Case 2A. $|k| \gtrsim\left|k_{i}\right|$ for $i=1,2,3$.

Symmetry allows us to assume $\left|k_{1}\right| \geq\left|k_{2}\right| \geq\left|k_{3}\right|$ and we must have $|k| \sim\left|k_{1}\right|$. Since $k=k_{1}+k_{2}+k_{3}$, we also have $\left|k^{3}-\left(k_{1}^{3}+k_{2}^{3}+k_{3}^{3}\right)\right| \lesssim\left|k k_{1} k_{2}\right|$ (see (4.2)). Therefore, in this case, the left side of (8.7) is bounded by

$$
\int_{*} \widehat{f}_{4}(k, \tau) \frac{\left|k k_{1} k_{2}\right|^{\frac{1}{2}}}{\langle k\rangle^{1-s} \prod_{i=1}^{3}\left\langle k_{i}\right\rangle^{s}\left\langle\tau_{i}-k_{i}^{3}\right\rangle^{\frac{1}{2}}} \widehat{f}_{1}\left(k_{1}, \tau_{1}\right) \widehat{f}_{2}\left(k_{2}, \tau_{2}\right) \widehat{f}_{3}\left(k_{3}, \tau_{3}\right) .
$$

Then, we may bound the preceding by

$$
\int_{*} \widehat{f}_{4}(k, \tau) \frac{\widehat{f_{2}}\left(k_{2}, \tau_{2}\right)}{\left\langle k_{2}\right\rangle^{s-\frac{1}{2}}\left\langle\tau_{2}-k_{2}^{3}\right\rangle^{\frac{1}{2}}} \frac{\widehat{f_{1}}\left(k_{1}, \tau_{1}\right)}{\left\langle\tau_{1}-k_{1}^{3}\right\rangle^{\frac{1}{2}}} \frac{\widehat{f_{3}}\left(k_{3}, \tau_{3}\right)}{\left\langle k_{3}\right\rangle^{s}\left\langle\tau_{3}-k_{3}^{3}\right\rangle^{\frac{1}{2}}}
$$

and (8.7) is equivalent to

$$
\left\|u_{1} u_{2} u_{3}\right\|_{L_{x, t}^{2}} \lesssim\left\|u_{1}\right\|_{X_{0, \frac{1}{2}}}\left\|u_{2}\right\|_{X_{s-\frac{1}{2}, \frac{1}{2}}}\left\|u_{3}\right\|_{X_{s, \frac{1}{2}}} .
$$

We recall from [5] that $X_{\delta, \frac{1}{2}} \subset L_{x, t}^{6}$ for any $\delta>0$. Therefore, we validate (8.8) using a Hölder application in $L_{x, t}^{4} L_{x, t}^{6} L_{x, t}^{12}$, with the required $L_{x, t}^{12}$ estimate given by Sobolev and the $L_{x, t}^{4}$ estimate from (7.31).

Case 2B. $|k| \ll\left|k_{i}\right|$ for $i=1,2,3$. 
We bound $\left|k^{3}-\left(k_{1}^{3}+k_{2}^{3}+k_{3}^{3}\right)\right| \lesssim\left|k_{1} k_{2} k_{3}\right|$ in this region and control the left side of (8.7) by

$$
\int_{*} \widehat{f}_{4}(k, \tau) \frac{1}{\langle k\rangle^{1-s} \prod_{i=1}^{3}\left\langle k_{i}\right\rangle^{s-\frac{1}{2}}\left\langle\tau_{i}-k_{i}^{3}\right\rangle^{\frac{1}{2}}} \widehat{f}_{1}\left(k_{1}, \tau_{1}\right) \widehat{f}_{2}\left(k_{2}, \tau_{2}\right) \widehat{f}_{3}\left(k_{3}, \tau_{3}\right) .
$$

If $s \in\left(\frac{1}{2}, 1\right]$, we may ignore $\frac{1}{\langle k\rangle^{1-s}}$ and finish things off with an $L_{x, t}^{2} L_{x, t}^{6} L_{x, t}^{6} L_{x, t}^{6}$ Hölder argument using $X_{\delta, \frac{1}{2}} \subset L_{x, t}^{6}$ for any $\delta>0$.

This completes the proof of (8.2) for $s \in\left(\frac{1}{2}, 1\right]$.

Remark 8.1. Bourgain has conjectured [5] that $X_{0, \frac{1}{2}} \subset L_{x, t}^{6}$. If this estimate were known, the previous discussion could be substantially simplified. Our proof of the $s=\frac{1}{2}$ case in [17] is partly motivated by an effort to prove this embedding estimate.

Lemma 8.2. If $m$ is of the form (4.7) with $s=-\frac{1}{2}$, then

$$
\left|\int_{0}^{\delta} \Lambda_{5}\left(M_{5}\right) d t\right| \lesssim \lambda^{0+} N^{-\frac{5}{2}+}\|I u\|_{Y^{0}}^{5} .
$$

The proof is a simple modification of the proof of Lemma 5.2 with (8.1) playing the role of (5.1). The projection appearing in the left side of (8.1) causes no trouble in this application, since, as shown in Lemma 4.6, $M_{5}$ vanishes when $k_{4}+k_{5}=0$ so it also vanishes when $k_{1}+k_{2}+k_{3}=0$. Note also that $-\frac{3}{4}+$ and $\frac{1}{4}-$ are systematically replaced by $-\frac{1}{2}$ and $\frac{1}{2}$ throughout the argument.

8.2. Rescaling. Our task is to construct the solution of the 1-periodic (7.1) on an arbitrary fixed time interval $[0, T]$. This is equivalent to showing the $\lambda$-rescaled problem with corresponding solution $u_{\lambda}(x, t)=\lambda^{-2} u\left(\frac{x}{\lambda}, \frac{t}{\lambda^{3}}\right)$ has a solution which exists on $\left[0, \lambda^{3} T\right]$. The lifetime of the variant local result is controlled by $\|I \phi\|_{L^{2}}$ and

We choose $\lambda$ so that

$$
\left\|I \phi_{\lambda}\right\|_{L^{2}(0, \lambda)} \lesssim \lambda^{-\frac{3}{2}-s} N^{-s}\|\phi\|_{H^{s}(0, \lambda)} .
$$

$$
\left\|I \phi_{\lambda}\right\|_{L^{2}(0, \lambda)}=\epsilon_{0} \ll 1 \Longrightarrow \lambda \sim N^{\frac{-s}{s+\frac{3}{2}}} .
$$

This choice guarantees that the local-in-time result for (7.1) is valid for a time interval of size 1 .

8.3. Almost conservation and iteration. The local result and Lemma 8.2 imply

$$
\left|E_{I}^{4}(1)-E_{I}^{4}(0)\right| \lesssim \lambda^{0+} N^{-\frac{5}{2}+} \epsilon_{0}^{5} .
$$

Recall that $\lambda=\lambda(N)$ so we may ignore $\lambda^{0+}$ by slightly adjusting $-\frac{5}{2}+$. Therefore, since by a (modification of) (6.6) $E_{I}^{4}(t) \sim\left\|I \phi_{\lambda}(t)\right\|_{L^{2}}^{2}$, we have that

$$
\left\|I \phi_{\lambda}(1)\right\|_{L^{2}}^{2}=\epsilon_{0}+C \epsilon_{0}^{5} N^{-\frac{5}{2}+}+O\left(\epsilon_{0}^{3}\right) .
$$

For small $\epsilon_{0}$ and large $N$ we see then that $\left\|I \phi_{\lambda}(1)\right\|_{L^{2}}$ is also of size $\epsilon_{0}$. We may iterate the local result $M$ times until, say, $E_{I}^{4}(M)$ first exceeds $2 E_{I}^{4}(0)$, that is, until

$$
M N^{-\frac{5}{2}+} \sim \epsilon_{0} \Longrightarrow M \sim N^{\frac{5}{2}-} .
$$

The solution of the $\lambda(N)$-periodic (7.1) is thus extended to the interval $\left[0, N^{\frac{5}{2}-}\right]$. We now choose $N=N(T)$ such that $N^{\frac{5}{2}-}>[\lambda(N)]^{3} T \sim N^{\frac{3}{2}} T$. This completes the proof that $(1.2)$ is globally well-posed in $H^{-\frac{1}{2}}(\mathbb{T})$. Comments similar to those 
presented in (6.19)- 6.26 apply to the periodic case showing that for our solution of (1.2) we have

$$
\|u(t)\|_{H^{-\frac{1}{2}}} \lesssim t^{\frac{1}{2}+}\|\phi\|_{H^{-\frac{1}{2}}}
$$

\section{Global Well-Posedness for MOdified KdV}

The results obtained for $\mathrm{KdV}$ are combined with some properties of the Miura transform 42] (see also the survey 43], 44]) to prove global well-posedness results for modified $\mathrm{KdV}(\mathrm{mKdV})$. This section contains the proofs of Theorems 3 and 4 . The initial value problem for $\mathbb{R}$-valued $\mathrm{mKdV}$ on the line is

$$
\left\{\begin{array}{c}
\partial_{t} u+\partial_{x}^{3} u \pm 6 u^{2} \partial_{x} u=0, \quad u: \mathbb{R} \times[0, T] \longmapsto \mathbb{R}, \\
u(0)=u_{0} .
\end{array}\right.
$$

The choice of sign distinguishes between the focussing $(+)$ and defocussing $(-)$ cases. This problem is known [31] to be locally well-posed in $H^{s}$ for $s \geq \frac{1}{4}$. The regularity requirement $s \geq \frac{1}{4}$ is sharp [33, [13. We establish global well-posedness of (9.1) in the range $s>\frac{1}{4}$ improving the work of Fonseca, Linares and Ponce [20].

9.1. Defocussing case. Consider the defocussing case of (9.1). The Miura transform of a solution $u$ is the function $v$ defined by

$$
v=M[u]=\partial_{x} u+u^{2} .
$$

A calculation shows that $v$ solves

$$
\left\{\begin{array}{c}
\partial_{t} v+\partial_{x}^{3} v-6 v \partial_{x} v=0, \quad v: \mathbb{R} \times[0, T] \longmapsto \mathbb{R} \\
v(0)=v_{0} .
\end{array}\right.
$$

Remark 9.1. Note that the uniqueness of the mKdV evolution is known [31 in a subspace of $C\left([0, T], H^{s}\right), s>\frac{1}{4}$, obtained by intersection in spaces associated with the maximal function and smoothing effect norms, while the KdV uniqueness holds in $X_{s-1, \frac{1}{2}+}$, a subspace of $C\left([0, T] ; H^{s-1}\right)$ which is not naturally identified within the image of $C\left([0, T] ; H^{s}\right)$ under the Miura transform. Nevertheless, the Miura image of the mKdV evolution coincides with the KdV evolution from an element of the Miura image. Let $S_{m K d V}(t)\left(u_{0}\right)$ denote the nonlinear solution flow map for the defocussing initial value problem (9.1) and let $S_{K d V}(t)\left(v_{0}\right)$ denote the flow map of (9.3). For smooth enough $v_{0}$, we have the intertwining relationship

$$
M\left[S_{m K d V}(t)\left(u_{0}\right)\right]=S_{K d V}(t)\left(M\left[u_{0}\right]\right) .
$$

Since the $\mathrm{KdV}$ evolution is uniquely determined in $X_{s-1, \frac{1}{2}+}$ for data in $H^{s-1}(\mathbb{R})$, the next lemma provides the regularity to show the Miura image of the mKdV evolution from $H^{s}, s>\frac{1}{4}$, data is the unique solution of $\mathrm{KdV}$. A similar remark applies to the focussing case.

Suppose the initial data $u_{0}$ for (9.1) is in $H^{s}, \frac{1}{4}<s<1$. We show that $v_{0}$ is in $H^{s-1}$.

Lemma 9.1. If $u_{0} \in H^{s}, \frac{1}{4}<s<1$, then $v_{0}=\partial_{x} u_{0}+u_{0}^{2} \in H^{s-1}$. 
Proof.

$$
\begin{aligned}
\left\|D^{s-1} v_{0}\right\|_{L^{2}} & \leq\left\|D^{s-1} \partial_{x} u_{0}\right\|_{L^{2}}+\left\|D^{s-1}\left(u_{0}^{2}\right)\right\|_{L^{2}} \\
& \leq\left\|D^{s} u_{0}\right\|_{L^{2}}+\left\|u_{0}^{2}\right\|_{L^{2}} \\
& \leq\left\|D^{s} u_{0}\right\|_{L^{2}}+\left\|u_{0}\right\|_{L^{4}}^{2} \\
& \lesssim\left\|D^{s} u_{0}\right\|_{L^{2}}+\left\|u_{0}\right\|_{H^{s}}^{2}
\end{aligned}
$$

The lemma verifies that the initial data $v_{0}$ for the $\mathrm{KdV}$ equation (9.3) is in $H^{s-1}$ and $-\frac{3}{4}<s-1$ since $\frac{1}{4}<s$. Therefore, the global well-posedness result for KdV just established applies to (9.3) and we know that the solution $v$ exists for all time and satisfies

$$
\|v(t)\|_{H^{s-1}} \lesssim(1+|t|)^{C}
$$

for some constant $C$. We exploit this polynomial-in-time bound for KdV solutions to control $\|u(t)\|_{H^{s}}$ using the Miura transform.

Note that

$$
\|u(t)\|_{H^{s}} \lesssim\|u(t)\|_{L^{2}}+\left\|\partial_{x} u(t)\right\|_{H^{s-1}} .
$$

Since our mKdV solution satisfies $L^{2}$-mass conservation, $\|u(t)\|_{L^{2}}=\left\|u_{0}\right\|_{L^{2}}$, it suffices to control $\left\|\partial_{x} u(t)\right\|_{H^{s-1}}$ to control $\|u(t)\|_{H^{s}}$. By (9.2),

$$
\left\|\partial_{x} u(t)\right\|_{H^{s-1}} \lesssim\|v(t)\|_{H^{s-1}}+\left\|u^{2}(t)\right\|_{H^{s-1}} .
$$

Using (9.5),

$$
\left\|\partial_{x} u(t)\right\|_{H^{s-1}} \lesssim(1+|t|)^{C}+\left\|u^{2}(t)\right\|_{H^{s-1}} .
$$

Summarizing, we have

$$
\|u(t)\|_{H^{s}} \lesssim 1+(1+|t|)^{C}+\left\|u^{2}(t)\right\|_{H^{s-1}} .
$$

Lemma 9.2. Assuming that $\|u(t)\|_{L^{2}} \lesssim 1$ and $\frac{1}{4}<s<1$, there exists an $\epsilon>0$ such that

$$
\left\|u^{2}(t)\right\|_{H^{s-1}} \lesssim\|u(t)\|_{H^{s}}^{1-\epsilon} .
$$

Assuming the lemma for a moment, observe that combining (9.7) and (9.6) implies a polynomial-in-time upper bound on $\|u(t)\|_{H^{s}}$ giving global well-posedness of defocussing mKdV. We now turn to the proof of (9.7).

Proof. We first consider the case when $\frac{1}{2}+\frac{1}{1000}<s<1$. The Sobolev estimate in one dimension

$$
\|w\|_{L^{q}} \lesssim\left\|D^{\sigma} w\right\|_{L^{p}}, \quad \frac{1}{q}=\frac{1}{p}-\frac{\sigma}{1}
$$

is applied with $w=D^{s-1}\left(u^{2}\right), \sigma=(1-s), q=2$, yielding for

$$
\frac{1}{p}=\frac{3}{2}-s
$$

that

$$
\left\|D^{s-1}\left(u^{2}\right)\right\|_{L^{2}} \lesssim\left\|u^{2}\right\|_{L^{p}}
$$

We continue the estimate by writing $\left\|u^{2}\right\|_{L^{p}}=\|u\|_{L^{2 p}}$ and using Sobolev to get

$$
\leq\|u\|_{L^{2 p}}^{2} \leq\|u\|_{H^{\sigma(p)}}^{2}, \quad \sigma(p)=\frac{1}{2}-\frac{1}{2 p} .
$$


Finally, we interpolate $H^{\sigma(p)}$ between $H^{0}=L^{2}$ and $H^{s}$ to obtain

$$
\left\|D^{s-1}\left(u^{2}\right)\right\|_{L^{2}} \lesssim\|u\|_{L^{2}}^{2(1-\theta)}\|u\|_{H^{s}}^{2 \theta}
$$

where $\theta=\frac{1}{s} \sigma(p)$. Using (9.9) and (9.10), we can simplify to find $\sigma(p)=\frac{2 s-1}{4}$ and $2 \theta=1-\frac{1}{2 s}$. Since $\|u\|_{L^{2}} \lesssim 1$, we observe that (9.7) holds in case $\frac{1}{2}+\frac{1}{1000}<s<1$.

In case $\frac{1}{4}<s \leq \frac{1}{2}+\frac{1}{1000}$, we begin with a crude step by writing

$$
\left\|D^{s-1}\left(u^{2}\right)\right\|_{L^{2}} \lesssim\left\|D^{s-\frac{2}{3}}\left(u^{2}\right)\right\|_{L^{2}} .
$$

Modifying the steps in the previous case, we have

$$
\left\|D^{s-\frac{2}{3}}\left(u^{2}\right)\right\|_{L^{2}} \leq\|u\|_{L^{2 p}}^{2}, \quad \frac{1}{p}=\frac{1}{2}+\frac{2}{3}-s .
$$

Then, by Sobolev and interpolation,

$$
\|u\|_{L^{2 p}}^{2} \lesssim\|u\|_{H^{\sigma(p)}}^{2} \lesssim\|u\|_{L^{2}}^{2(1-\theta)}\|u\|_{H^{s}}^{2 \theta}
$$

where

$$
\theta=\frac{1}{s} \sigma(p), \sigma(p)=\frac{1}{2}-\frac{1}{2 p}=\frac{s}{2}-\frac{1}{12}
$$

It is then clear that for $s \in\left(\frac{1}{4}, \frac{1}{2}+\frac{1}{1000}\right]$, we have $2 \theta=1-\frac{1}{6 s}=1-\epsilon$ for an appropriate $\epsilon>0$ as claimed.

9.2. Focussing case. In the focussing case of $\mathbb{R}$-valued modified $\mathrm{KdV}$, the Miura transform has a different form:

$$
v=\partial_{x} u+i u^{2} .
$$

The function $v$ solves the complex $\mathrm{KdV}$ initial value problem

$$
\left\{\begin{array}{c}
\partial_{t} v+\partial_{x}^{3} v-i 6 v \partial_{x} v=0, \quad v: \mathbb{R} \times[0, T] \longmapsto \mathbb{C}, \\
v(0)=v_{0}
\end{array}\right.
$$

Since the solution $u(t)$ of focussing modified $\mathrm{KdV}$ is $\mathbb{R}$-valued and derivatives are more costly than squaring in one dimension, we take the perspective that $v$ is "nearly $\mathbb{R}$-valued". The variant local result for 9.12 has an existence interval determined by $\left(\int\left|I v_{0}\right|^{2} d x\right)^{\frac{1}{2}}$. However, (9.12) does not conserve $\left(\int|I v(t)|^{2} d x\right)^{\frac{1}{2}}$ but instead (almost) conserves $\left|\int(I v(t))^{2} d x\right|^{\frac{1}{2}}$. An iteration argument showing global well-posedness may proceed if we show that

$$
\left|\int(I v(t))^{2} d x\right|^{\frac{1}{2}} \text { controls }\left(\int|I v(t)|^{2} d x\right)^{\frac{1}{2}}
$$

for functions $v$ of the form given by the Miura transform (9.11).

Observe that

$$
\begin{gathered}
\left(\int|I v|^{2} d x\right)^{\frac{1}{2}}=\left(\int\left(I u_{x}\right)^{2}+\left(I\left(u^{2}\right)\right)^{2} d x\right)^{\frac{1}{2}} \\
\left|\int(I v)^{2} d x\right|^{\frac{1}{2}}=\left|\int\left(I u_{x}\right)^{2}-\left(I\left(u^{2}\right)\right)^{2} d x+2 i \int\left(I u_{x}\right)\left(I\left(u^{2}\right)\right) d x\right|^{\frac{1}{2}} .
\end{gathered}
$$


Lemma 9.3. Assuming that $\|u(t)\|_{L^{2}} \leq C$ for all $t$, and $\frac{1}{4}<s<1$, there exists an $\epsilon>0$ such that

$$
\left\|I\left(u^{2}\right)\right\|_{L^{2}} \leq \epsilon\left\|I u_{x}\right\|_{L^{2}}+C
$$

If we take the lemma for granted, we deduce from (9.13) and (9.14) that

$$
\begin{aligned}
\left.\left.\left|\int\right| I v(t)\right|^{2} d x\right|^{\frac{1}{2}} \text { is bounded } & \Longleftrightarrow\left(\int(I v(t))^{2} d x\right)^{\frac{1}{2}} \text { is bounded } \\
& \Longleftrightarrow\left|\int\left(I u_{x}(t)\right)^{2} d x\right|^{\frac{1}{2}} \text { is bounded. }
\end{aligned}
$$

The equivalence (9.16) links the quantity determining the length of the local existence interval to an almost conserved quantity. Consequently, (9.12) is GWP and $\left|\int(I v(t))^{2} d x\right|^{\frac{1}{2}}$, is polynomially bounded in $t$. Since $\|u(t)\|_{L^{2}} \leq C$ for solutions of focussing modified $\mathrm{KdV}$, the equivalence (9.16) implies $\|u(t)\|_{H^{s}}, s>\frac{1}{4}$, is polynomially bounded in $t$. Therefore, focussing $\mathrm{mKdV}$ is globally well-posed in $H^{s}(\mathbb{R}), s>\frac{1}{4}$, provided we prove the lemma above.

Proof of Lemma 9.3. We use duality and rewrite the expression to be controlled as

$$
\int f\left(\xi_{1}+\xi_{2}\right) m\left(\xi_{1}+\xi_{2}\right) \widehat{u_{1}}\left(\xi_{1}\right) \widehat{u_{2}}\left(\xi_{2}\right) d \xi_{1} d \xi_{2}
$$

where $m(\xi) \sim 1$ when $|\xi| \lesssim N$ and $m(\xi) \sim N^{-(s-1)}|\xi|^{s-1}$ when $|\xi|>N$ and we have relaxed to the bilinear situation. The function $f$ is introduced to calculate the norm using duality so $\|f\|_{L^{2}} \leq 1$. We may assume that $\widehat{u_{j}}$ is nonnegative. Symmetry allows us to assume $\left|\xi_{1}\right| \geq\left|\xi_{2}\right|$.

Case 1. $\left|\xi_{1}\right| \lesssim N \Longrightarrow m\left(\xi_{1}+\xi_{2}\right) \sim 1$.

In this case, $I$ acts like the identity operator and the task is to control $\|u\|_{L^{4}}^{2}$. By Sobolev and interpolation,

$$
\|u\|_{L^{4}}^{2} \leq\|u\|_{L^{2}}\|u\|_{\dot{H}^{\frac{1}{2}}} \leq\|u\|_{L^{2}}^{\frac{3}{2}}\|u\|_{\dot{H}^{1}}^{\frac{1}{2}}
$$

and we observe that, in this case,

$$
\left\|I\left(u^{2}\right)\right\|_{L^{2}} \leq C+\epsilon\left\|I\left(\partial_{x} u\right)\right\|_{L^{2}} .
$$

Case 2. $\left|\xi_{1}\right| \gg N$.

Case 2A. $\left|\xi_{1}+\xi_{2}\right| \lesssim N \Longrightarrow\left|\xi_{1}\right| \sim\left|\xi_{2}\right|, m\left(\xi_{1}+\xi_{2}\right) \sim 1$.

We decompose the factors dyadically by writing

$$
\int f\left(\xi_{1}+\xi_{2}\right) \widehat{u_{1}}\left(\xi_{1}\right) \widehat{u_{2}}\left(\xi_{2}\right) d \xi_{1} d \xi_{2} \sim \sum_{N_{1} \sim N_{2} \gg N} \int f\left(\xi_{1}+\xi_{2}\right) \widehat{u_{N_{1}}}\left(\xi_{1}\right) \widehat{u_{N_{2}}}\left(\xi_{2}\right) d \xi_{1} d \xi_{2} .
$$

We focus on a particular dyadic interaction term

$$
\begin{aligned}
& \int_{\left\{\left|\xi_{i}\right| \sim N_{i}\right\}} f\left(\xi_{1}+\xi_{2}\right) \widehat{u_{N_{1}}}\left(\xi_{1}\right) \widehat{u_{N_{2}}}\left(\xi_{2}\right) d \xi_{1} d \xi_{2} \\
& \quad \sim \int_{\left\{\left|\xi_{i}\right| \sim N_{i}\right\}} f\left(\xi_{1}+\xi_{2}\right) m\left(\xi_{1}\right) \xi_{1} \widehat{u_{N_{1}}}\left(\xi_{1}\right) \frac{1}{m\left(\xi_{1}\right) \xi_{1}} \widehat{u_{N_{2}}}\left(\xi_{2}\right) d \xi_{1} d \xi_{2} .
\end{aligned}
$$


In the Case $2 \mathrm{~A}$ region, $m\left(\xi_{1}\right) \xi_{1} \sim N_{1}^{s} N^{-(s-1)}$. We make this substitution and apply Cauchy-Schwarz in $\xi_{2}$ to observe

$$
\lesssim\|f\|_{L_{\{|\xi| \lesssim N\}}^{2}}\left\|\widehat{u_{N_{2}}}\right\|_{L^{2}} \frac{1}{N_{1}^{s}}\left\|\left|\xi_{1}\right|^{s} \widehat{u_{N_{1}}}\left(\xi_{1}\right)\right\|_{L^{1}}
$$

Multiplying through by $1=\frac{N^{-(s-1)}}{N^{-(s-1)}}$ leads to

$$
\begin{aligned}
& \lesssim\|f\|_{L^{2}}\left\|\widehat{u_{N_{2}}}\right\|_{L^{2}} \frac{N^{s-1}}{N_{1}^{s-\frac{1}{2}}}\left\|I \partial_{x} u_{N_{1}}\right\|_{L^{2}} \\
& \ll\left\|I \partial_{x} u_{N_{1}}\right\|_{L^{2}}, \text { provided } \frac{1}{2}<s<1,
\end{aligned}
$$

since $N_{1}>N \gg 1$. Of course we can sum over the dyadic scales and retain the claim.

It remains to establish the claim for the Case $2 \mathrm{~A}$ region when $\frac{1}{4}<s \leq \frac{1}{2}$. We rewrite the expression (9.17) differently as

$$
\int \frac{f\left(\xi_{1}+\xi_{2}\right)}{\left(1+\left|\xi_{1}+\xi_{2}\right|\right)^{\frac{1}{2}}+}\left(1+\left|\xi_{1}+\xi_{2}\right|\right)^{\frac{1}{2}+}\left|\xi_{1}\right|^{s} \widehat{u_{N_{1}}}\left(\xi_{1}\right) \frac{1}{\left|\xi_{1}\right|^{s}} \widehat{u_{N_{2}}}\left(\xi_{2}\right) d \xi_{1} d \xi_{2}
$$

Defining $\widehat{D}(\xi)=\frac{f\left(\xi_{1}+\xi_{2}\right)}{\left(1+\left|\xi_{1}+\xi_{2}\right|\right)^{\frac{1}{2}+}}, \widehat{U_{N_{1}}}\left(\xi_{1}\right)=\left|\xi_{1}\right|^{s} \widehat{u_{N_{1}}}\left(\xi_{1}\right)$, we observe that the preceding expression is controlled by

$$
N^{\frac{1}{2}+} N_{1}^{-s}\left\langle\widehat{D}, \widehat{U_{N_{1}}} * \widehat{u_{N_{2}}}\right\rangle
$$

We apply Hölder to estimate by

$$
N^{\frac{1}{2}+} N_{1}^{-s}\|D\|_{L^{\infty}}\left\|U_{N_{1}}\right\|_{L^{2}}\left\|u_{N_{2}}\right\|_{L^{2}}
$$

and Sobolev implies $\|D\|_{L^{\infty}} \leq\|f\|_{L^{2}}$. Rewriting this gives

$$
N^{\frac{1}{2}}+\frac{1}{N_{1}^{s}}\|d\|_{L^{2}}\left\|\left|\xi_{1}\right|^{s} \widehat{u_{N_{1}}}\left(\xi_{1}\right)\right\|_{L^{2}}\left\|u_{N_{2}}\right\|_{L^{2}}
$$

Multiplying by $\frac{N^{-(s-1)}}{N^{-(s-1)}}$ yields

$$
\left(\frac{N}{N_{1}}\right)^{s} \frac{1}{N^{\frac{1}{2}}-}\|f\|_{L^{2}}\left\|u_{N_{2}}\right\|_{L^{2}}\left\|I \partial_{x} u_{N_{1}}\right\|_{L^{2}}
$$

and the prefactor vanishes as $N \rightarrow \infty$, proving the claimed estimate.

Case 2B. $\left|\xi_{1}+\xi_{2}\right| \gg N$.

We multiply (9.17) through by $m\left(\xi_{1}\right) \xi_{1}$ in numerator and denominator. We observe that $\frac{m\left(\xi_{1}+\xi_{2}\right)}{m\left(\xi_{1}\right)} \lesssim 1$ and use the argument passing through $L_{\xi_{1}}^{1}$ in Case $2 \mathrm{~A}$ to complete the proof.

9.3. Modified KdV on $\mathbb{T}$. Lemmas 9.2 and 9.3 naturally extend to the $\lambda$-periodic setting. These results link the polynomial-in-time upper bound (8.10) for solutions of the $\lambda$-periodic initial value problem (7.1) for $\mathrm{KdV}$ to a polynomial-in-time upper bound on $\|u(t)\|_{H^{\frac{1}{2}(\mathbb{T})}}$ for solutions of the $\lambda$-periodic initial value problem for mKdV, implying Theorem 4. 


\section{REFERENCES}

[1] M. Beals. Self-spreading and strength of singularities for solutions to semilinear wave equations. Ann. of Math. (2), 118(1):187-214, 1983. MR 85c:35057

[2] B. Birnir, C. Kenig, G. Ponce, N. Svanstedt, L. Vega. On the ill-posedness of the Initial Value Problem for the generalizedKorteweg-de Vries and nonlinear Schrödinger equations. $J$. London Math. Soc. (2). 53(3):551-559, 1996. MR 97d:35233

[3] B. Birnir, G. Ponce, N. Svanstedt. The local ill-posedness of the modified KdV equation. Ann. Inst. H. Poincaré Anal. Non-Linéaire. 13(4):529-535, 1996. MR 97e:35152

[4] J. L. Bona and R. Smith. The initial-value problem for the Korteweg-de Vries equation. Philos. Trans. Roy. Soc. London Ser. A, 278(1287):555-601, 1975. MR 52:6219

[5] J. Bourgain. Fourier transform restriction phenomena for certain lattice subsets and applications to nonlinear evolution equations I, II. Geom. Funct. Anal., 3:107-156, 209-262, 1993. MR 95d:35160a, MR 95d:35160b

[6] J. Bourgain. Approximation of solutions of the cubic nonlinear Schrödinger equations by finite-dimensional equations and nonsqueezing properties. Internat. Math. Res. Notices, 2:7988, 1994. MR 95f:35237

[7] J. Bourgain. Aspects of long time behaviour of solutions of nonlinear Hamiltonian evolution equations Geom. Funct. Anal., 5:105-140, 1995. MR 96f:35151

[8] J. Bourgain. On the growth in time of higher Sobolev norms of smooth solutions of Hamiltonian PDE. Internat. Math. Res. Notices, 6:277-304, 1996. MR 97k:35016

[9] J. Bourgain. Periodic Korteweg de Vries equation with measures as initial data. Selecta Math. (N.S.), 3(2):115-159, 1997. MR 2000i:35173

[10] J. Bourgain. Refinements of Strichartz' inequality and applications to 2D-NLS with critical nonlinearity. International Mathematical Research Notices, 5:253-283, 1998. MR 99f:35184

[11] L. Carleson and P. Sjölin. Oscillatory integrals and a multiplier problem for the disc. Studia Math., 44:287-299. (errata insert), 1972. Collection of articles honoring the completion by Antoni Zygmund of 50 years of scientific activity, III. MR 50:14052

[12] A. Cohen. Existence and regularity for solutions of the Korteweg-de Vries equation. Arch. Rational Mech. Anal., 71(2):143-175, 1979. MR 80g:35109

[13] M. Christ, J. Colliander, and T. Tao Asymptotics, frequency modulation and low regularity ill-posedness for canonical defocusing equations. To appear Amer. J. Math., 2002.

[14] J. Colliander, M. Keel, G. Staffilani, H. Takaoka, and T. Tao. A refined global wellposedness result for Schrödinger equations with derivative. To appear SIAM J. Math. Anal., 2002.

[15] J. Colliander, M. Keel, G. Staffilani, H. Takaoka, and T. Tao. Global well-posedness for $\mathrm{KdV}$ in Sobolev spaces of negative index. Electron. J. Diff. Eqns., 2001(26):1-7, 2001. MR 2001m:35269

[16] J. Colliander, M. Keel, G. Staffilani, H. Takaoka, and T. Tao. Global wellposedness for Schrödinger equations with derivative. SIAM Journal of Mathematical Analysis, 2001. SIAM J. Math. Anal. 33(3):649-669, 2001. MR 2002j:35278

[17] J. Colliander, M. Keel, G. Staffilani, H. Takaoka, and T. Tao. Multilinear estimates for periodic KdV equations and applications. To appear J. Funct. Anal., 2002.

[18] J. E. Colliander, G. Staffilani, and H. Takaoka. Global Wellposedness of KdV below $l^{2}$. Mathematical Research Letters, 6(5,6):755-778, 1999. MR 2000m:35159

[19] C. Fefferman. A note on spherical summation multipliers. Israel J. Math., 15:44-52, 1973. MR 47:9160

[20] G. Fonseca, F. Linares, and G. Ponce. Global well-posedness for the modified Kortewegde Vries equation. Comm. Partial Differential Equations, 24(3-4):683-705, 1999. MR 2000a:35210

[21] G. Fonseca, F. Linares, and G. Ponce. Global existence for the critical generalized KdV equation. Preprint, 2002.

[22] J. Ginibre. An introduction to nonlinear Schrödinger equations. In Nonlinear waves (Sapporo, 1995), pages 85-133. Gakkōtosho, Tokyo, 1997. MR 99a:35235

[23] J. Ginibre, Y. Tsutsumi, and G. Velo. Existence and uniqueness of solutions for the generalized Korteweg de Vries equation. Math. Z., 203(1):9-36, 1990. MR 90m:35168

[24] J. Ginibre. Le problème de Cauchy pour des EDP semi-linéaires périodiques en variables d'espace (d'après Bourgain). Astérisque, 237:Exp. No. 796, 4, 163-187, 1996. Séminaire Bourbaki, Vol. 1994/95. MR 98e:35154 
[25] A. Grünrock. A bilinear Airy-estimate with application to gKdV-3. Preprint, 2001.

[26] H. Hofer and E. Zehnder. Symplectic invariants and Hamiltonian dynamics. Birkhäuser Verlag, Basel, 1994. MR 96g:58001

[27] J.-L. Joly, G. Métivier, and J. Rauch. A nonlinear instability for $3 \times 3$ systems of conservation laws. Comm. Math. Phys., 162(1):47-59, 1994. MR 95f:35145

[28] T. Kato. The Cauchy problem for the Korteweg-de Vries equation. Nonlinear partial differential equations and their applications. Collège de France Seminar, Vol. I (Paris, 1978/1979), pages 293-307. Pitman, Boston, Mass., 1981. MR 82m:35129

[29] M. Keel and T. Tao. Local and Global Well-Posedness of Wave Maps on $\mathbb{R}^{1+1}$ for Rough Data. International Mathematical Research Notices, 21:1117-1156, 1998. MR 99k:58180

[30] M. Keel and T. Tao. Global well-posedness for large data for the Maxwell-Klein-Gordon equation below the energy norm. Preprint, 2000.

[31] C. Kenig, G. Ponce, and L. Vega. Well-Posedness and Scattering Results for the Generalized Korteweg-de Vries Equation via the Contraction Principle. Communications on Pure and Applied Mathematics, XLVI:527-620, 1993. MR 94h:35229

[32] C. E. Kenig, G. Ponce, and L. Vega. A bilinear estimate with applications to the KdV equation. J. Amer. Math. Soc., 9:573-603, 1996. MR 96k:35159

[33] C. E. Kenig, G. Ponce, and L. Vega. On the ill-posedness of some canonical dispersive equations. Duke Math. J., 106(3):617-633, 2001. MR 2002c:35265

[34] C. E. Kenig, G. Ponce, and L. Vega. Global well-posedness for semi-linear wave equations. Comm. Partial Differential Equations, 25(9-10):1741-1752, 2000. MR 2001h:35128

[35] S. B. Kuksin. On squeezing and flow of energy for nonlinear wave equations. Geom. Funct. Anal., 5:668-711, 1995. MR 96d:35091

[36] S. B. Kuksin. Infinite-dimensional symplectic capacities and a squeezing theorem for Hamiltonian PDEs. Comm. Math. Phys. 167(3):531-552, 1995. MR 96e:58060

[37] S. Klainerman and M. Machedon. Smoothing estimates for null forms and applications. Internat. Math. Res. Notices, 9, 1994. MR 95i:58174

[38] Y. Martel and F. Merle. Blow up in finite time and dynamics of blow up solutions for the $L^{2}$ critical generalized KdV equation. J. Amer. Math. Soc. 15(3):617-664, 2002.

[39] F. Merle. Personal communication. 2002.

[40] Y. Meyer and R. R. Coifman. Ondelettes et opérateurs. III. Hermann, Paris, 1991. Opérateurs multilinéaires. [Multilinear operators]. MR 93m:42004]

[41] Y. Meyer and R. Coifman. Wavelets. Calderón-Zygmund and multilinear operators, translated from the 1990 and 1991 French originals by David Salinger, Cambridge University Press, Cambridge, 1997. MR 98e:42001

[42] R. M. Miura. Korteweg-de Vries equation and generalizations. I. A remarkable explicit nonlinear transformation. J. Mathematical Phys., 9:1202-1204, 1968. MR 40:6042a

[43] R. M. Miura. The Korteweg-de Vries Equation: A Survey of Results. SIAM Review, 18(3):412 - 459, 1976. MR 53:8689

[44] R. M. Miura. Errata: "The Korteweg-deVries equation: a survey of results" (SIAM Rev. 18 (1976), no. 3, 412-459). SIAM Rev., 19(4):vi, 1977. MR 57:6908

[45] K. Nakanishi, H. Takaoka, and Y. Tsutsumi. Counterexamples to bilinear estimates related to the KdV equation and the nonlinear Schrödinger equation. Methods of Appl. Anal. 8(4):569$578,2001$.

[46] J. Rauch and M. Reed. Nonlinear microlocal analysis of semilinear hyperbolic systems in one space dimension. Duke Math. J., 49:397-475, 1982. MR 83m:35098

[47] R. R. Rosales. I. Exact solution of some nonlinear evolution equations, II. The similarity solution for the Korteweg-de Vries equation and the related Painlevé transcendent. PhD thesis, California Institute of Technology, 1977.

[48] H. Takaoka. Global Well-posedness for the Kadomtsev-Petviashvili II Equation. Discrete Cont. Dynam. Systems. 6(2):483-499, 1999. MR 2000m:35163

[49] T. Tao. Multilinear weighted convolution of $L^{2}$ functions and applications to nonlinear dispersive equations. Amer. J. Math., 123(5):839-908, 2001. MR 2002k:35283

[50] N. Tzvetkov. Global low regularity solutions for Kadomtsev-Petviashvili equation. Differential Integral Equations., 13(10-12):1289-1320, 2001. MR 2001g:35227 
Department of Mathematics, University of Toronto, Toronto, On Canada, M5S 3G3

School of Mathematics, University of Minnesota, Minneapolis, Minnesota, 55455

Department of Mathematics, Stanford University, Stanford, California 94305-2125

Current address: Department of Mathematics, Massachusetts Institute of Technology, 77 Massachusetts Avenue, Cambridge, Massachusetts 02138

Department of Mathematics, Hokkaido University, Sapporo 060-0810, Japan

Current address: Department of Mathematics, Kobe University, Rokko, Kobe 657-8501, Japan

Department of Mathematics, University of California, los Angeles, Los Angeles, CAlifornia 90095-1555 